\title{
Gut Microbiota Participates in Antithyroid Drug Induced Liver Injury Through the Lipopolysaccharide Related Signaling Pathway
}

\author{
Jiayu Sun ${ }^{\dagger}$, Fuya Zhao ${ }^{\dagger}$, Baiqiang Lin ${ }^{\dagger}$, Jing Feng, Xin Wu, Yang Liu, Lei Zhao, Biqiang Zhu
} and Yunwei Wei *

Department of Oncology and Laparoscopy Surgery, The First Affiliated Hospital of Harbin Medical University, Harbin, China

OPEN ACCESS

Edited by:

Raffaele Capasso,

University of Naples Federico II, Italy

Reviewed by:

Jasenka Zubcevic,

University of Florida, United States

Daiqiong Fang,

Zhejiang University, China

*Correspondence:

Yunwei Wei

hydwyw11@hotmail.com

${ }^{t}$ These authors have contributed equally to this work

Specialty section:

This article was submitted to Gastrointestinal and Hepatic

Pharmacology,

a section of the journal

Frontiers in Pharmacology

Received: 28 August 2020 Accepted: 16 November 2020

Published: 17 December 2020

Citation:

Sun J, Zhao F, Lin B, Feng J, Wu X, Liu Y, Zhao L, Zhu B and Wei Y (2020)

Gut Microbiota Participates in Antithyroid Drug Induced Liver Injury

Through the Lipopolysaccharide

Related Signaling Pathway.

Front. Pharmacol. 11:598170.

doi: 10.3389/fphar.2020.598170
Background: Drugs can alter the gut microbiota structure, and gut microbiota dysbiosis in turn is correlated with drug side effects through the intestinal endotoxemia hypothesis. Whether antithyroid drugs (including methimazole and propylthiouracil) cause gut microbiota dysbiosis and whether the gut microbiota is correlated with antithyroid drugs induced liver injury is unknown.

Methods: Initial Graves' disease patients were randomly divided into the methimazole group ( $n=20)$ and the propylthiouracil group $(n=20)$ and were followed up every 2 weeks; 50 healthy controls were also included. The structure and function of gut microbiota were compared from the cross sectional and longitudinal levels. The correlation between the gut microbiota and clinical parameters was also determined. In addition, Sprague-Dawley rats were randomly allotted into six groups, including four drug groups, which received daily doses of methimazole ( $1.5 \mathrm{mg} / \mathrm{kg} /$ day; $2.5 \mathrm{mg} / \mathrm{kg} /$ day) or propylthiouracil $(7.5 \mathrm{mg} / \mathrm{kg} / \mathrm{day}$; $12.5 \mathrm{mg} / \mathrm{kg} /$ day) by oral gavage, and two control groups received the vehicle. In addition to the indexes mentioned above, intestinal barrier-related indexes were also performed.

Results: Cross sectional and longitudinal comparison results from both clinical trials and animal studies indicate that antithyroid drugs altered gut microbiota structure; and the liver function related indexes all increased which correlated with gut microbiota. In addition, lipopolysaccharide-related pathways and the lipopolysaccharide concentration in feces and serum all increased after antithyroid drugs administration. These results consistent with the destroyed intestinal barrier in animal study after antithyroid drugs administration.

Conclusion: We verified that antithyroid drugs altered gut microbiota structure and that the gut microbiota may in turn be correlated with antithyroid drugs-induced liver injury through the intestinal endotoxemia hypothesis.

Keywords: gut microbiota, antithyroid drugs, lipopolysaccharide, intestinal barrier, liver injury

\section{INTRODUCTION}

Antithyroid drugs (ATDs), including methimazole (MMI) and propylthiouracil (PTU), represent the first-line treatment in Europe, Asia and Latin America (Bartalena et al., 2016; Brito et al., 2016). Liver injury is a side effect of ATDs treatment, although hyperthyroidism itself can lead to different degrees of liver damage (Li et al., 2015). In a study of drug-induced liver injury (DILI) across 308 medical 
centers in China, MMI, and PTU each accounted for one among 72 death cases (Shen et al., 2019). PTU treatment was even reported to be the third greatest cause of drug-related liver transplantation in the United States (1992-2002) (Russo et al., 2004). However, the specific mechanisms of ATDs-induced liver injury have not yet been fully elucidated, although the cytotoxic substance glyoxal, which is the intermediate product of MMI metabolism, can induce oxidative stress and cell dysfunction (Heidari et al., 2015).

The gut microbiota, a hidden functional organ, has important roles in modulating physiological functions, including immune development and maturation, hematopoiesis and metabolism regulation (Manzo and Bhatt, 2015; Enright et al., 2016; Cani, 2018). Meanwhile, the gut microbiota can be affected by many factors, such as age, diet and drugs. Maier et al. reported that $24 \%$ of synthetic drugs with human targets across therapeutic classes inhibited the growth of at least one bacterial strain in vitro (Maier et al., 2018). Furthermore, some synthetic drugs antibiotics (Zarrinpar et al., 2018), proton pump inhibitors (PPIs) (Jackson et al., 2016), chemotherapeutics (Montassier et al., 2016), metformin (Wu et al., 2017), and antidepressants (Morgan et al., 2014), altered the composition of the gut microbiota in vivo during disease treatment. In turn, the gut microbiota can also metabolize some synthetic drugs. On the one hand, the gut microbiota can modulate the expression of some host cytochrome enzymes, such as CYP8b1 and CYP3a11, which can metabolize drugs (Li et al., 2016). On the other hand, the gut microbiota can directly produce some enzymes that participate in drug absorption, activation and inactivation, which may be closely related to the toxic side effects of drugs (Li and Jia, 2013). The bidirectional drug-microbiome interactions will inevitably contribute to the toxic side effects of many other drugs.

Through intensive studies, the gut microbiota has also been shown to participate in DILI via the following main mechanisms. 1) Drug metabolism: dysbiosis of the gut microbiota induced by a drug in turn leads to drug metabolism into toxic liver metabolites through direct or indirect regulation of the secretion of some enzymes, such as myeloperoxidase and cytochrome P450 (CYP450) (Romano et al., 2015; Aziz et al., 2018). 2) The intestinal endotoxemia hypothesis: a drug may give rise to gut microbiota dysbiosis, intestinal barrier injury and increased intestinal permeability, thus permitting gut microbiota or microbiota-derived product, such as lipopolysaccharide (LPS), translocation into the portal circulation and then transit to the liver. Then intestinal-derived LPS binds to toll-like receptor-4 (TLR-4) on hepatic sinusoidal endothelial cells and Kupffer cells, which can directly or indirectly lead to liver damage; and the gut microbiota transported to the liver can also activate a large number of hepatic inflammatory cells, which synthesize and release many proinflammatory factors in response to membrane antigens or toxic metabolites (such as LPS), causing further liver damage (Chassaing et al., 2014; Gong et al., 2019).

Previous studies have found that gut microbiota in initial Graves' disease (GD) patients (Init_GD group) differed from that in healthy controls (HCs) though the differential gut microbiota is not exactly the same (Zhou et al., 2014; Ishaq et al., 2018); and some drug could alter the composition of the gut microbiota in vivo during disease treatment as mentioned above, ATDs may also cause changes in gut microbiota. Hence, we postulated that the intestinal endotoxemia hypothesis participates in ATDs-induced liver injury.

In clinical treatment, ATDs are often accompanied by adjuvant drugs, such as hepatoprotective drugs and leukogenic drugs, which may also affect the gut microbiota structure. Therefore, to eliminate the effect of hyperthyroidism and adjuvant drugs on the gut microbiota, we designed clinical and animal studies to 1) observe changes in the gut microbiota structure caused by ATDs (including the use or nonuse of ATDs, different ATDs, different ATD doses and different time points) and 2) to preliminarily investigate the roles of the gut microbiota in ATDs-induced liver injury, with the hope of identifying a novel therapeutic strategy to prevent it.

\section{MATERIALS AND METHODS}

\section{Ethics Statement, Informed Consented and Clinical Trial Registration}

The ethics protocols of the clinical (Eth. 201815) and animal studies (Eth. 2018021) used in this study were all approved by the Ethics Committee of the First Affiliated Hospital of Harbin Medical University in accordance with the relevant regulations and laws. All participants were informed of the nature of the study and provided written informed consent. In addition, a clinical study was registered with the America Clinical Trial Registry (NCT 03433352).

\section{Recruitment of Init_GD Patients and Healthy Controls}

We recruited 40 Init_GD patients from the Endocrine Clinic of the First Affiliated Hospital of Harbin Medical University between December 2017 and December 2018. All Init_GD patients were randomly divided into MMI group $(n=20)$ and PTU group $(n=20)$ and were followed up every two weeks during the first month of ATDs treatment (Treat_GD group). The inclusion criteria were as follows: 18-65 years of age and taking medicine according to prescription. During the same time period, 50 age-, sex- and BMI-matched HCs were recruited from the Health Screening Center (Supplementary Figure S1), and all the participants met the same exclusion criteria (provided in the Supplementary Material).

\section{Sample Collection and Clinical Parameters}

Blood and fecal samples were collected from all Init_GD patients at first diagnosis and 2 and 4 weeks after ATDs administration; as well as HCs. The specific collection and processing methods and a comprehensive description of the clinical parameter analysis are provided in the supplementary information. Blood samples were collected for thyroid function detection (fT3, fT4 and thyroidstimulating hormone $[\mathrm{TSH}]$ ) and thyroid autoantibody (Tg-Ab and TPO-Ab), biochemical and serum LPS analyses; fecal samples were used for 16S rRNA gene sequence and fecal LPS detection. 
11 pairs of samples failed to be collected, left 159 pairs of samples that were used for further analysis in this study. The samples included 60 pairs from MMI group and 49 pairs from PTU group and 50 pairs of samples from HCs.

\section{Animals}

Forty-five female SPF Sprague-Dawley (SD) rats (6-8 weeks old, weighing $200 \pm 10 \mathrm{~g}$ ) were purchased from the animal experiment center of the Second Affiliated Hospital of Harbin Medical University. The rats were housed in a temperature- and humidity-maintained room $\left(20 \pm 3^{\circ} \mathrm{C}\right.$, with a relative humidity of approximately $40 \%$ ) under a $12 \mathrm{~h}$ light/dark cycle in stainless steel cages with free access to water and standard rat chow. The rats were allowed to acclimate to their diet and new environment for at least 1 week before initiating the experiment.

\section{Experimental Setup}

Rats were randomly allotted to six groups, including four ATDs groups ( $n=9$ for each group), which received daily doses of $1.5 \mathrm{mg} / \mathrm{kg}$ /day MMI (ML group), $2.5 \mathrm{mg} / \mathrm{kg} /$ day MMI (MH group), $7.5 \mathrm{mg} / \mathrm{kg} /$ day PTU (PL group) or $12.5 \mathrm{mg} / \mathrm{kg} /$ day PTU (PH group) by oral gavage. The two control groups ( $n=4$ for Control $1 ; n=5$ for Control 2) received the same doses of vehicle (ordinary drinking water) every day. Control two group rats were introduced later and raised for only 2 weeks to match their body weight with that of the ATDs group because the original control one rats had a heavier body weight, which may affect the gut microbiota structure and intestinal barrier. The study lasted for 11 weeks (the experimental design and timeline naming of the rats are shown in Supplementary Figure S2).

\section{Sample Collection and Basic Characteristic Measurements}

Blood samples were collected for thyroid function detection (fT3, fT4, and TSH) and biochemical and serum LPS analyses. Fecal samples were used for 16S rRNA gene sequence, fecal LPS and fecal calprotectin (CALP) detection. Related tissue samples (including the thyroid, liver, spleen, cecum and total gastrointestinal tract) were also collected. Intestines (including small intestine and colon) electron microscopy analyses, which all correlate with intestinal barrier, were performed (Fang et al., 2018). Thirty-seven fecal samples failed to be collected due to death of some rats; thus, 123 fecal samples were analyzed in this study. The specific sample collection and measurement methods are described in the supplementary information.

\section{Statistical Analyses}

The laboratory examination and analysis of basic animal indicators were performed using the Statistical Package for the Social Sciences (SPSS) version 22.0 (SPSS Inc., 2010 Chicago, IL, United States); mean and corresponding standard error of the mean (SEM) values were calculated, the Wilcoxon rank-sum test was performed as an alternative when data were not normally distributed and Kruskal-Wallis $\mathrm{H}$ test was performed when it involved three or more groups. Other specific methods are presented in the supplementary information.

\section{RESULTS}

\section{Part 1: Clinical Study Study Population and Clinical Parameters}

All 90 participants were of Han nationality, born in northeastern China and had a similar dietary structure. The Init_GD, Treat_GD and HCs groups were all age- $(p=0.838)$, sex- $(p=0.934)$ and BMI-matched $(p=0.563)$ (Table 1). With ATDs use, hyperthyroidism of Init_GD patients gradually eased, fT3 and fT4 levels decreased even the TSH level remained low, and Tg-Ab and TPO-Ab levels showed decreasing trends, while the TPO-Ab level showed no significant difference between the Init_GD and Treat_GD groups $(p=0.319$, Supplementary Table S1). Indicators of liver function, such as ALT, AST and AKP, were higher in both the Init_GD and Treat_GD groups than in the HCs $(p<0.0001$ among the three indicators), which is consistent with the changes reported in a previous study regarding hyperthyroidism-related liver injury (Li et al., 2015); the Treat_GD group showed more significant differences than the Init_GD group, although ALT and AST levels showed no significant differences between them ( $p=$ 0.858 and $p=0.687)$. Furthermore, the fecal supernatant LPS, CALP and serum LPS levels showed higher levels in the Init_GD and Treat_GD groups than in the HC group $(p<0.0001$ among all three indicators). The specific clinical characteristics of GD patients and HCs are shown in Table 1 and Supplementary Table S1.

\section{Gut Microbial Structure Analysis Sequencing Characteristics}

From the 159 samples, 12, 346, 125 high-quality sequences were selected based on barcode- and primer-sequence filtering, with an average of 77,649 (ranging from 50,651 to 92,227) sequences per barcoded sample. A total of 3,442 OTUs were delineated at a $97 \%$ similarity level. The rarefaction curve of all samples reached a plateau (Supplementary Figure S3A), and Good's coverage for all samples was greater than $99.3 \%$, indicating a satisfactory gut microbiota sequencing depth. Furthermore, we divided these samples into 11 subgroups according to the aims of our research; the specific information is shown in Supplementary Table S2. The aims were as follows:

\section{Part 1: To Investigate Whether Antithyroid Drugs Could Cause Changes in the Gut Microbiota Structure (Treat_GD vs. Init_GD vs. Healthy Control; Treat_GD vs. Init_GD; Treat_GD vs. Healthy Control; Init_GD vs. Healthy Control) \\ Alpha and Beta Diversity}

First, we compared the gut microbiota alpha diversity of the three groups (Init_GD, Treat_GD and HC). The Ace index increased in the Init_GD and Treat_GD groups compared with that in the HC group, and the Treat_GD group showed more higher (Init_GD vs. $\mathrm{HC}, p=7.5 \mathrm{e}-03$; Treat_GD vs. $\mathrm{HC}, p=3.3 \mathrm{e}-06$, Figure 1A). Similarly, the Shannon index was also higher in Treat_GD group 
TABLE 1 | Clinical and demographic features of GD patients and healthy controls.

\begin{tabular}{|c|c|c|c|c|c|c|c|c|c|c|c|c|}
\hline \multirow[t]{3}{*}{ Parameters } & \multicolumn{6}{|c|}{ Init_GD + Treat_GD } & \multirow[t]{3}{*}{$\mathrm{HC}$} & \multirow[t]{3}{*}{ p1 } & \multirow[t]{3}{*}{ p2 } & \multirow[t]{3}{*}{ p3 } & \multirow[t]{3}{*}{ p4 } & \multirow[t]{3}{*}{ p5 } \\
\hline & \multicolumn{4}{|c|}{ MMI } & \multicolumn{2}{|c|}{ PTU } & & & & & & \\
\hline & MO & M2 & M4 & PO & P2 & P4 & & & & & & \\
\hline Age (year, mean $\pm S D$ ) & $36.1 \pm 12.15$ & $36.1 \pm 12.15$ & $36.1 \pm 12.15$ & $39.3 \pm 9.5$ & $37.82 \pm 10.97$ & $40.22 \pm 9.58$ & $36.58 \pm 9.06$ & 0.838 & 0.925 & 0.289 & 1 & 0.819 \\
\hline SEX (F/M) & $17 / 3$ & $17 / 3$ & $17 / 3$ & $16 / 4$ & $7 / 4$ & $13 / 5$ & $40 / 10$ & 0.934 & 0.722 & 0.111 & 1 & 0.607 \\
\hline $\mathrm{BMI}\left(\mathrm{kg} / \mathrm{m}^{2}\right.$, mean $\left.\pm \mathrm{SD}\right)$ & $21.89 \pm 2.94$ & $22.23 \pm 2.88$ & $22.59 \pm 2.89$ & $22.24 \pm 3.09$ & $22.12 \pm 2.69$ & $23.02 \pm 3.19$ & $21.83 \pm 2.13$ & 0.563 & 0.379 & 0.539 & 0.633 & 0.484 \\
\hline Rash & & $4 / 16$ & & & $3 / 17$ & & $0 / 50$ & & & 0.173 & & \\
\hline fT3 (pg/mL) & $20 / 0$ & $19 / 1$ & $13 / 7$ & $20 / 0$ & $11 / 0$ & $18 / 0$ & $0 / 50$ & 0 & 0.026 & 0.017 & 0.004 & 1 \\
\hline fT4 (ng/dl) & $20 / 0$ & $16 / 4$ & $12 / 8$ & $20 / 0$ & $9 / 2$ & $14 / 4$ & $0 / 50$ & 0 & 0 & 0.385 & 0.004 & 0.05 \\
\hline TSH (ulU/mL) & $20 / 0$ & $20 / 0$ & $19 / 1$ & $20 / 0$ & $11 / 0$ & $18 / 0$ & $0 / 50$ & 0 & 1 & 1 & 1 & 1 \\
\hline Tg-Ab (IU/ml) & $18 / 2$ & $12 / 8$ & $13 / 7$ & $18 / 2$ & $6 / 5$ & $14 / 4$ & $0 / 50$ & 0 & 0.004 & 0.578 & 0.087 & 0.09 \\
\hline TPO-Ab (IU/ml) & $18 / 2$ & $18 / 2$ & $18 / 2$ & $17 / 3$ & $10 / 1$ & $15 / 3$ & $0 / 50$ & 0 & 1 & 0.712 & 1 & 1 \\
\hline WBC (109/L) & $2 / 18$ & $2 / 18$ & $3 / 17$ & $0 / 20$ & $0 / 11$ & $1 / 17$ & $0 / 50$ & 0.146 & 1 & 0.389 & 1 & 0.592 \\
\hline NEUT (109/L) & $2 / 18$ & $2 / 18$ & $3 / 17$ & $1 / 19$ & $0 / 11$ & $2 / 16$ & $0 / 50$ & 0.048 & 0.743 & 0.69 & 1 & 0.599 \\
\hline LYMPH(109/L) & $0 / 20$ & $0 / 20$ & $1 / 19$ & $0 / 20$ & $0 / 11$ & $0 / 18$ & $0 / 50$ & 1 & 1 & 1 & 1 & 1 \\
\hline MONO(109/L) & $0 / 20$ & $0 / 20$ & $0 / 20$ & $0 / 20$ & $0 / 11$ & $0 / 18$ & $0 / 50$ & 1 & 1 & 1 & 1 & 1 \\
\hline EO (109/L) & $0 / 20$ & $0 / 20$ & $0 / 20$ & $0 / 20$ & $0 / 11$ & $0 / 18$ & $0 / 50$ & 1 & 1 & 1 & 1 & 1 \\
\hline BASO(109/L) & $0 / 20$ & $0 / 20$ & $0 / 20$ & $0 / 20$ & $0 / 11$ & $0 / 18$ & $0 / 50$ & 1 & 1 & 1 & 1 & 1 \\
\hline $\mathrm{RBC}(1012 /)$ & $2 / 18$ & $5 / 15$ & $4 / 16$ & $5 / 15$ & $3 / 8$ & $6 / 12$ & $0 / 50$ & 0 & 0.352 & 0.579 & 0.589 & 0.919 \\
\hline HCT (\%) & $0 / 20$ & $0 / 20$ & $0 / 20$ & $0 / 20$ & $1 / 10$ & $0 / 18$ & $0 / 50$ & 1 & 1 & 1 & 1 & 0.224 \\
\hline $\operatorname{MCV}(f L)$ & $11 / 9$ & $10 / 10$ & $11 / 9$ & $8 / 12$ & $0 / 11$ & $8 / 10$ & $0 / 50$ & 0 & 0.689 & 0.05 & 1 & 0.02 \\
\hline $\mathrm{MCH}(\mathrm{pg})$ & $7 / 13$ & $7 / 13$ & $9 / 11$ & $3 / 17$ & $0 / 11$ & $2 / 16$ & $0 / 50$ & 0 & 1 & 0.002 & 0,842 & 0.521 \\
\hline $\mathrm{MCHC}(\mathrm{g} / \mathrm{L})$ & $4 / 16$ & $3 / 17$ & $4 / 16$ & $0 / 20$ & $0 / 11$ & $1 / 17$ & $0 / 50$ & 0.025 & 1 & 0.126 & 1 & 0.592 \\
\hline РCT (\%) & $0 / 20$ & $0 / 20$ & $0 / 20$ & $0 / 20$ & $0 / 11$ & $0 / 18$ & $0 / 50$ & 1 & 1 & 1 & 1 & 1 \\
\hline MPV(fL) & $0 / 20$ & $0 / 20$ & $0 / 20$ & $0 / 20$ & $0 / 11$ & $0 / 18$ & $0 / 50$ & 1 & 1 & 1 & 1 & 1 \\
\hline HGB (g/L) & $1 / 19$ & $1 / 19$ & $0 / 20$ & $1 / 19$ & $3 / 8$ & $2 / 16$ & $0 / 50$ & 0.075 & 0.265 & 0.076 & 1 & 0.163 \\
\hline PLT (109/L) & $4 / 16$ & $7 / 13$ & $6 / 14$ & $3 / 17$ & $3 / 8$ & $2 / 16$ & $0 / 50$ & 0 & 0.352 & 0.154 & 0.675 & 0.544 \\
\hline $\mathrm{ALT}(\mathrm{U} / \mathrm{L})$ & $8 / 12$ & $10 / 10$ & $7 / 13$ & $3 / 17$ & $2 / 9$ & $2 / 16$ & $0 / 50$ & 0 & 0.829 & 0.011 & 0.72 & 0.881 \\
\hline AST (U/L) & $1 / 19$ & $6 / 14$ & $4 / 16$ & $1 / 19$ & $1 / 10$ & $0 / 18$ & $0 / 50$ & 0.003 & 0.127 & 0.019 & 0.15 & 0.694 \\
\hline GGT (U/L) & $3 / 17$ & $1 / 19$ & $5 / 15$ & $7 / 13$ & $4 / 7$ & $4 / 14$ & $0 / 50$ & 0 & 0.634 & 0.199 & 0.265 & 0.623 \\
\hline $\mathrm{TP}(\mathrm{g} / \mathrm{L})$ & $1 / 19$ & $2 / 18$ & $2 / 18$ & $1 / 19$ & $0 / 11$ & $1 / 17$ & $0 / 50$ & 0.146 & 1 & 0.389 & 1 & 1 \\
\hline ALB (g/L) & $0 / 20$ & $0 / 20$ & $0 / 20$ & $0 / 20$ & $0 / 11$ & $0 / 18$ & $0 / 50$ & 1 & 1 & 1 & 1 & 1 \\
\hline GLB (g/L) & $1 / 19$ & $4 / 16$ & $5 / 15$ & $5 / 15$ & $4 / 7$ & $3 / 15$ & $0 / 50$ & 0 & 0.335 & 0.874 & 0.305 & 0.528 \\
\hline $\mathrm{AKP}(\mathrm{U} / \mathrm{L})$ & $7 / 13$ & $8 / 12$ & $11 / 9$ & $12 / 8$ & $7 / 4$ & $14 / 4$ & $0 / 50$ & 0 & 0.323 & 0.038 & 0,515 & 0.466 \\
\hline TBIL (umol/L) & $2 / 18$ & $0 / 20$ & $0 / 20$ & $5 / 15$ & $3 / 8$ & $5 / 13$ & $0 / 50$ & 0.005 & 0.401 & 0.001 & 0.322 & 1 \\
\hline DBIL (umol/L) & $7 / 13$ & $2 / 18$ & $5 / 15$ & $8 / 12$ & $4 / 7$ & $7 / 11$ & $0 / 50$ & 0 & 0.28 & 0.056 & 0.207 & 1 \\
\hline IBIL (umol/L) & $2 / 18$ & $0 / 20$ & $1 / 19$ & $5 / 15$ & $3 / 8$ & $5 / 13$ & $0 / 50$ & 0.004 & 0.58 & 0.003 & 0.766 & 1 \\
\hline TBA (umol/L) & $1 / 19$ & $1 / 19$ & $0 / 20$ & $0 / 20$ & $0 / 11$ & $0 / 18$ & $0 / 50$ & 0.725 & 1 & 1 & 1 & 1 \\
\hline PA (mg/L) & $10 / 10$ & $4 / 16$ & $4 / 16$ & $7 / 13$ & $1 / 10$ & $2 / 16$ & $0 / 50$ & 0 & 0.003 & 0.336 & 0.087 & 0.161 \\
\hline
\end{tabular}

The measurement data and numeration data were statistically analyzed with $t$ test (or one-way ANOVA for multi group comparison) and $\chi^{2}$ test, respectively. Measurement data are expressed as the mean $\pm S D$ and numeration data are expressed as rate or composition (n1/n2 means abnormal/normal). F/M, female/male; BMI, body mass index; fT3, free triiodothyronine; fT4, free thyroxine; TSH, thyroid-stimulating hormone; Tg-Ab, antithyroglobulin antibody; TPO-Ab, antithyroperoxidase antibody; WBC, white blood cell; NEUT, neutrophils; LYMPH, lymphocytes; MONO, monocytes; EO, eosinophils; BASO, basophils; RBC, red blood cell; HCT, hematocrit; MCV, mean corpuscular volume; $\mathrm{MCH}$, mean corpuscular hemoglobin; MCHC, mean corpuscular hemoglobin concentration; PCT, platelet crit; MPV, mean platelet volume; HGB, hemoglobin; PLT, platelet; $A L T$, alanine aminotransferase; AST, aspartate transaminase; GGT, gamma-glutamyl transpeptidase; TP, total protein; ALB, albumin; GLB, globulin; AKP, alkaline phosphatase; TBIL, total bilirubin; DBIL, direct bilirubin; IBIL, indirect bilirubin; TBA, total bile acid; PA, pre-albumin and SD, standard deviation. Init GD: including MO and PO; Treat GD: including MMI and PTU; p1: $p$ Value of Init_GD vs. Treat_GD vs. HC; p2: p Value of Init_GD vs. Treat_GD; $p 3: p$ Value of MMI vs. PTU; $p 4: p$ Value of M0 vs. M2 vs. M4; $p 5$ : p Value of PO vs. P2 vs. P4.

than in the Init_GD group though there was no significant difference between them. However, both the two groups was lower in the Init_GD and Treat_GD groups than in the HC group (Init_GD vs. HC, $p=0.047$; Treat_GD vs. $\mathrm{HC}, p=0.83$, Figure 1B). In addition, the other alpha diversity indexes are shown in Supplementary Table S2.

To evaluate the similarities and differences among microbiota communities, PCoA was performed, which illustrated that the Init_GD and Treat_GD groups both deviated from the HC group. The Treat_GD group showed a greater deviation from the HC group than the Init_GD group ( $p=0.001$, Figure $1 C)$, and the Treat_GD group partially deviated from the Init_GD group when compared separately ( $p=0.064$, Supplementary Figure S3B).

The alpha and beta diversity results all indicated that the gut microbiota structures of the Init_GD and Treat_GD groups differed from that of the $\mathrm{HC}$ group and that the gut microbiota structure of the Treat_GD group differed from that of the Init_GD group (i.e., the gut microbiota varied after ATDs treatment), although the difference was not as obvious as those between the GD groups and the $\mathrm{HC}$ group. 


\section{Differentially Abundant Species Analysis}

To identify the specific communities associated with ATDs treatment, we further compared the gut microbiota structure between the Treat_GD and Init_GD groups; 11 species, representing four families $(p<0.05$, Supplementary Figure S3C) and 7 genera $(p<0.05$, Figure 1D), were significantly differentiated. Faecalibacterium and Clostridium_sensu_stricto_1 showed lower abundance in the Treat_GD group than in the Init_GD group, while Eubacterium_rectale, Romboutsia and Dorea showed higher abundance in the Treat_GD group at the genus level. Then, we compared the gut microbiota structure of the Init_GD and Treat_GD groups with that of the HC group, and 40 differentially abundant genera were revealed in the Treat_GD group vs. the HC group. Thirtyone differentially abundant genera were revealed in the Init_GD group vs. the $\mathrm{HC}$ group, and 30 of them were also differentially abundant between the Treat_GD group and the HC group (Supplementary Figure S3D). Most of the 30 differentially abundant species showed an increasing or decreasing trend after ATDs treatment based on the change in the Init_GD group compared with the HC group, which may be because the previous the gut microbiota structure changes were aggravated by medication (Figure 1D). For example, Bacteroides abundance was significantly decreased in the Init_GD and Treat_GD groups compared with that in the HC group, and that in the Treat_GD group decreased more, though the difference showed no statistical significance between the Treat_GD and Init_GD groups. Similarly, Blautia abundance showed the opposite trend, which illustrated that the gut microbiota structure in the Init_GD group differed from that in the HC group and that Blautia abundance in the Treat_GD group showed more deterioration (Figure 1D).

In the present study, 44 differentially abundant species, representing two phyla, 11 families $(p<0.05$, Supplementary Figure S3C) and 31 genera $(p<0.05$, Figure 1D), were revealed between the Init_GD group and the HC group. However, the differentially abundant species were not exactly the same among different studies. For example, at the phylum level, our present and previous study both showed that the Actinobacteria and Firmicutes proportions were increased while the Bacteroidetes proportion was decreased in Init_GD patients compared with those in HCs (Supplementary Figure S3C). In addition, an animal study on Graves' ophthalmopathy (GO) also showed higher Firmicutes and lower Bacteroidetes abundance in the GO group than in the control group (Masetti et al., 2018), while two other clinical studies on hyperthyroidism and GO showed higher Bacteroidetes and lower Firmicutes abundances (Ishaq et al., 2018; Shi et al., 2019).

The microbial dysbiosis index (MDI, the MDI is defined as the $\log$ of [total abundance in genera increased in disease group] over [total abundance of genera decreased in disease group]) and the Firmicutes/Bacteroidetes ratio $(\mathrm{F} / \mathrm{B}$ ratio) both indicate gut microbiota disturbance, and the $\mathrm{F} / \mathrm{B}$ ratio is even considered to be representative of health status (Turnbaugh et al., 2006; Gevers et al., 2014). Similar to the change in the alpha diversity Ace index, both of these indexes were significantly higher in the Init_GD and Treat_GD groups than in the HC group, and these indexes were more higher in the Treat_GD group and significantly correlated with PC1 of the PCoA (MDI, $r=-0.741, p=6.2 \mathrm{e}-29$; F/B ratio, $r=$ $-0.624, p=1.6 \mathrm{e}-18$ ) (Figure 1E; Supplementary Figure S3E). These results showed that dysbiosis occurred in the Init_GD and Treat_GD groups, and the Treat_GD group showed greater disturbances than the Init_GD group.

\section{Part 2: To Investigate Whether Different Antithyroid Drugs Cause Different Gut Microbiota Structure Changes (Methimazole (Including M2 and M4) vs. Propylthiouracil (Including P2 and P4) vs. Healthy Control; Methimazole vs. Propylthiouracil; Methimazole vs. Healthy Control; Propylthiouracil vs. Healthy Control)}

It is well known that the pharmacodynamics and toxicology differ between the MMI and PTU, and whether these differences are correlated with the gut microbiota is unknown (Franklyn, 2009). Therefore, we first performed a holistic analysis of the gut microbiota between the MMI, PTU and $\mathrm{HC}$ groups and then performed detailed comparisons at each specific time point.

\section{Alpha and Beta Diversity}

The gut microbiota Ace index was significantly increased in the MMI group compared with that in the PTU group ( $p=1.9 \mathrm{e}-04)$, and both the MMI and PTU groups showed higher Ace indexes than the HC group (MMI vs. HC, $p=3.2 \mathrm{e}-09$; PTU vs. HC, $p=0.17$; Figure 1F). The Shannon index was not significantly different among the three groups $(p=0.32)$. Interestingly, the MMI group showed a lower Shannon index, while the PTU group showed a higher Shannon index than the HC group (MMI vs. HC, $p=0.37$; PTU vs. HCs $p=0.47$; Figure 1G). The other alpha diversity indexes are shown in Supplementary Table S2. PCoA also revealed differences in the distributions of the three groups $(p=0.001)$ : the PTU group was more similar to the HC group than the MMI group (Figure 1H), and the MMI group significantly deviated from the PTU group when they were compared separately $(p=0.026$, Supplementary Figure S4A).

\section{Differentially Abundant Species Analysis}

Both alpha and beta diversity results showed that the gut microbiota structure in the MMI group differed from that in the PTU group. We then compared the gut microbiota composition of the MMI and PTU groups, and 25 discriminative species, representing two phyla, six families $(p<0.05$, Supplementary Figure S4B), and 17 genera $(p<$ 0.05 , Figure 1I), were revealed. At the phylum level, a higher proportion of Firmicutes was shown in the MMI group than in the PTU group, while Bacteroidetes was more prevalent in the PTU group. The corresponding family, Enterobacteriaceae, was more prevalent in the MMI group, whereas Bacteroidaceae was more prevalent in the PTU group. At the genus level, Blautia and Escherichia-Shigella were enriched in the MMI group, while 


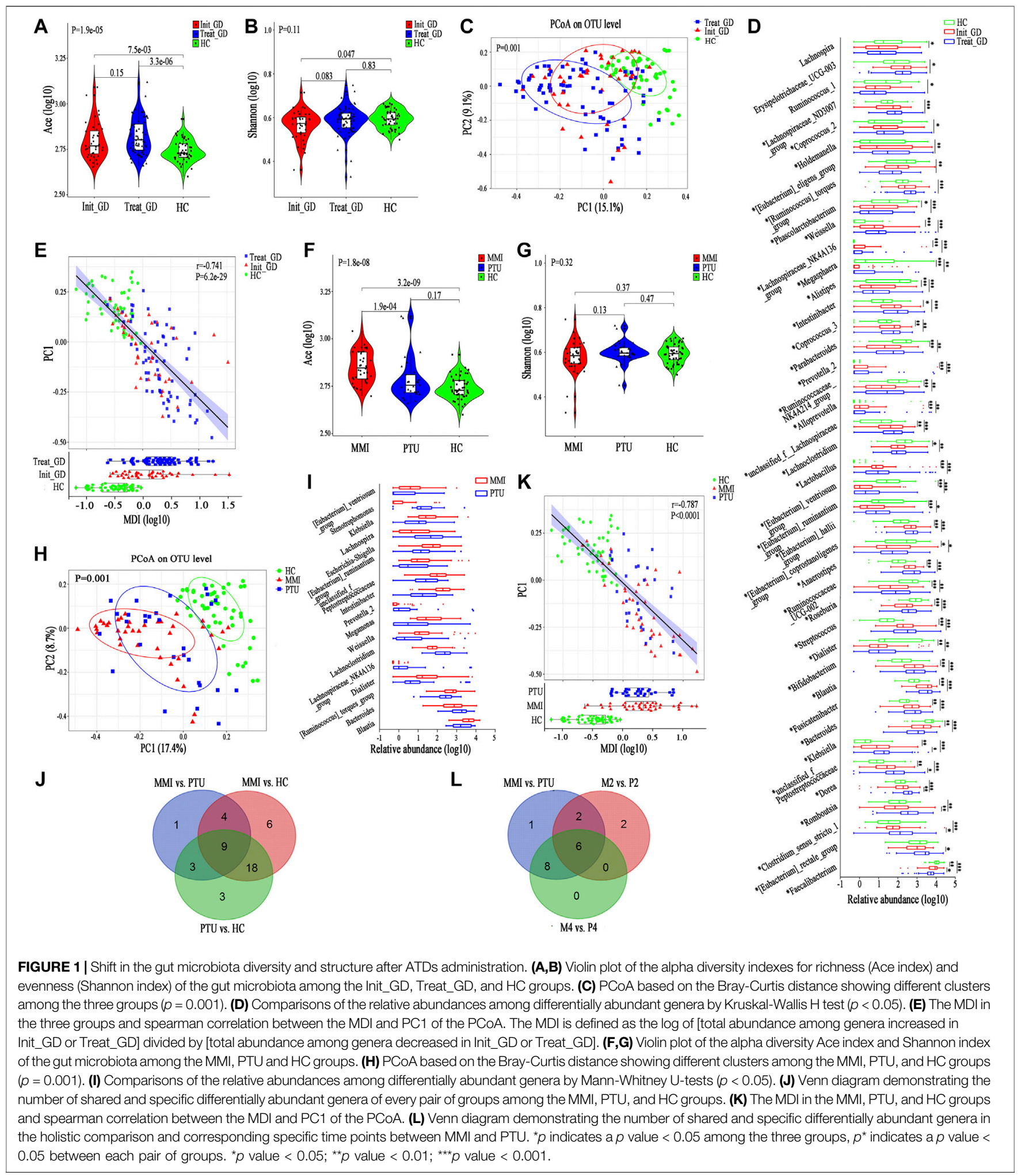

Bacteroides, Lachnospiraceae_NK4A136_group and Lachnoclostridium were enriched in the PTU group. To further illustrate the different effects of different ATDs on the gut microbiota structure, we compared the gut microbiota of the
MMI and PTU groups with that of the HC group, and the differentially abundant species among the groups are shown in a Venn diagram. An additional six differentially abundant genera were unique in MMI vs. those in $\mathrm{HC}$, and three genera were 
unique in PTU vs. those in $\mathrm{HC}$, which further reflects the difference in the microbiota between the MMI and PTU groups (Figure 1J; Supplementary Table S3). Similarly, the $\mathrm{MDI}$ and $\mathrm{F} / \mathrm{B}$ ratio changes were also consistent with the gut microbiota changes. Both the MDI and F/B ratio were higher in the MMI and PTU groups than in the HC group, and the MMI group exhibited a higher MDI and F/B ratio than the PTU group. Additionally, the two indexes correlated with PC1 (MDI, $r=-0.787, p<0.0001$, Figure $1 \mathrm{~K} ; \mathrm{F} / \mathrm{B}$ ratio, $r=-0.651, p=1.15 \mathrm{e}-15$, Supplementary Figure S4C), which showed that the gut microbiota structure was destroyed in both the MMI and PTU groups and that the MMI group showed greater dysbiosis than the PTU group.

Part 3: To Investigate the Longitudinal Changes in the Gut Microbiota Structure Caused by Antithyroid Drugs at Different Time Points

As shown above, the ATDs and different ATDs all contributed to an altered gut microbiota structure, and our previous study showed that the gut microbiota partially recovered in euthyroid GD patients compared with that in Init_GD patients. However, the short-term longitudinal changes in the gut microbiota structure during drug treatment (whether they become more disordered and then normalize or they gradually normalize) are unknown. We compared the gut microbiota changes in the MMI group and the PTU group over the time course of treatments.

\section{LONGITUDINAL CHANGES IN THE GUT MICROBIOTA STRUCTURE DURING METHIMAZOLE TREATMENT (MO VS. M2 VS. M4 VS. HEALTHY CONTROL; MO VS. M2 VS. M4 (INCLUDING SEPARATE COMPARISONS OF EACH PAIR OF GROUPS))}

\section{Alpha and Beta Diversity}

The Ace index was significantly higher in the M0, M2 and M4 groups than in the HC group (M0 vs. HC, $p=2.6 \mathrm{e}-6$; $\mathrm{M} 2$ vs. $\mathrm{HC}$, $p=2.3 \mathrm{e}-6 ; \mathrm{M} 4$ vs. $\mathrm{HC}, \mathrm{p}=2 \mathrm{e}-6)$, although no significant differences were found among the M0, M2 and M4 groups. The Ace index increased at the 2nd week after taking MMI, but interestingly, it declined at the 4 th week, which may be because of the partial recovery of thyroid function (Figure 2A). The Shannon index also showed a similar trend among the M0, M2 and M4 groups, although no significant differences were found among groups or in comparison with the HC group ( $p=0.35$ ). Nonetheless, the M0 group showed a lower Shannon index than the HC group, which is consistent with a lower alpha diversity in Init_GD patients than in HCs (Figure 2B; Supplementary Table S2). Consistent with the alpha diversity results, PCoA showed that the M0, M2, and M4 groups clearly deviated from the HC group ( $p=0.001)$; the M2 and M4 groups were farther from the $\mathrm{HC}$ group than the M0 group; while the M2 and M4 groups were more similar to one another (Figure 2C).
The other alpha diversity indexes are shown in Supplementary Table S2.

\section{Differentially Abundant Species Analysis}

Differentially abundant species analysis was also performed to explore differences among different time points (M0-M2-M4), which included comparisons between each pair of time points. Only six discriminative features (representing one family and five genera) (Supplementary Figure S4D) with a prevalence $>10 \%$ and a maximum proportion $>0.2 \%$ were found. However, as we mentioned above, PCoA showed that the M0, M2, and M4 groups deviated from one another, which may be because of the low abundance of differentially abundant species playing a dominant role. In truth, an additional 20 discriminative features, representing four families and 16 genera, with a prevalence $>10 \%$ (low-abundance microbiota constituents), are also shown in Supplementary Figures S4E, F. Among them, the abundance of microbiota constituents Romboutsia and Clostridium_sensu_stricto_1 increased, while Ruminococcaceae_UCG-013 abundance decreased, according to a comparison among the three time points (Figures 2D-I). Some genera, such as Faecalibacterium and Blautia, had no statistical significance in comparisons among the three time points (M0 vs. M2 vs. M4) but were significantly different from those in HCs and showed longitudinal changes (Figures 2J-L).

\section{LONGITUDINAL CHANGES IN THE GUT MICROBIOTA STRUCTURE DURING PROPYLTHIOURACIL TREATMENT (PO VS. P2 VS. P4 VS. HEALTHY CONTROL; PO VS. P2 VS. P4, INCLUDING SEPARATE COMPARISONS OF EACH PAIR OF GROUPS)}

\section{Alpha and Beta Diversity}

The alpha diversity longitudinal change trend in the PTU group was similar to that in the MMI group among the P0, P2 and $\mathrm{P} 4$ time points (increased at the 2 nd week and decreased at the 4th week); however, the Ace and Shannon indexes showed no significant differences among the P0, P2 and P4 time points compared with those of HCs except for an increased Ace index at P2 (Ace: P2 vs. HC, $p=0.029$; P2 vs. P0, $p=0.025$; Figures $\mathbf{2 M}, \mathbf{N})$. Consistent with the reduced alpha diversity in Init_GD patients, the two indexes were lower at P0 than in HCs. PCoA also showed that $\mathrm{P} 0, \mathrm{P} 2$, and $\mathrm{P} 4$ clearly deviated from HCs $(p=0.001$, Figure 2O). P2 and P4 were farther from HCs than P0 and P2 was the furthest from HCs.

\section{Differentially Abundant Species Analysis}

Differentially abundant species analysis among the three time points (P0-P2-P4) showed only two discriminative species (Eubacterium_rectale and Dorea) with a prevalence $>10 \%$ and a maximum proportion $>0.2 \%$, and both species showed increasing trends over the time of PTU use (Figures 2P,Q; 

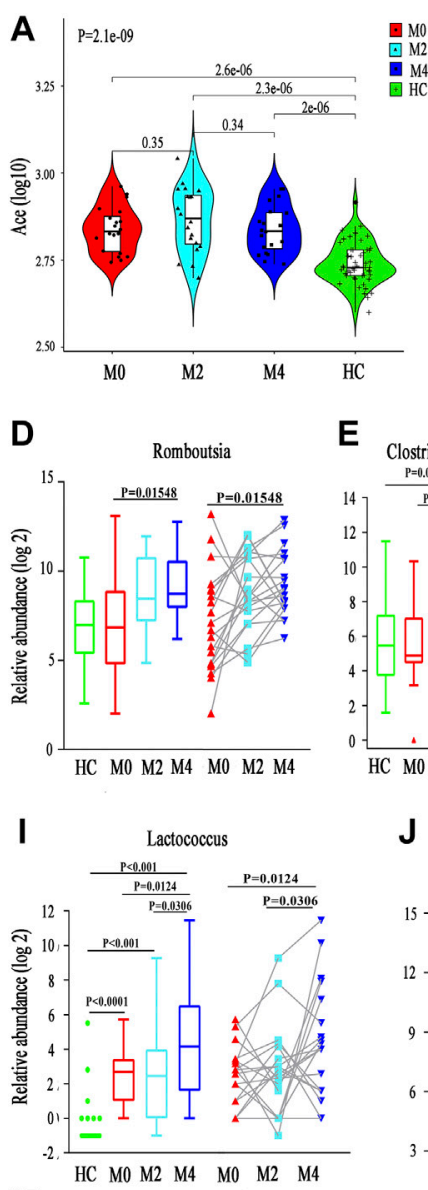

M
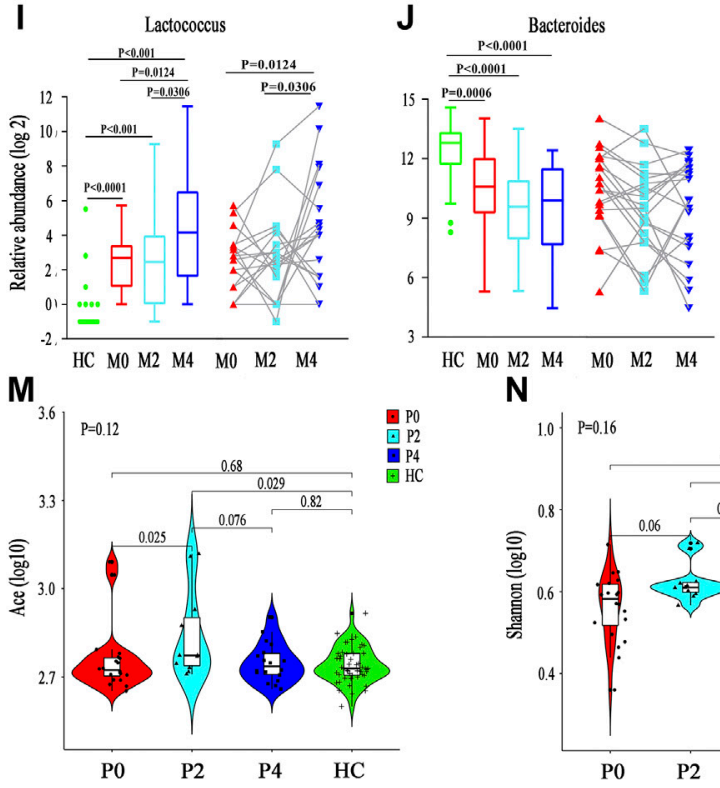

$\mathbf{N}$

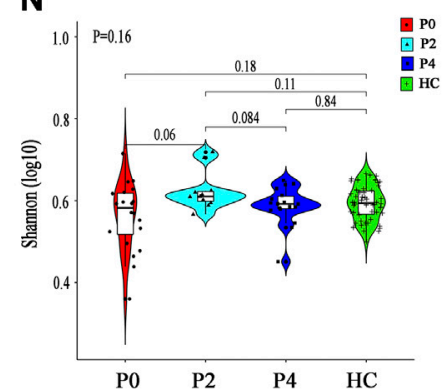

E Clostridium_sensu_stricto_1
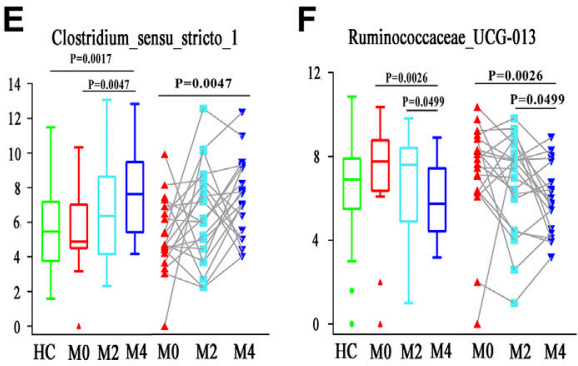

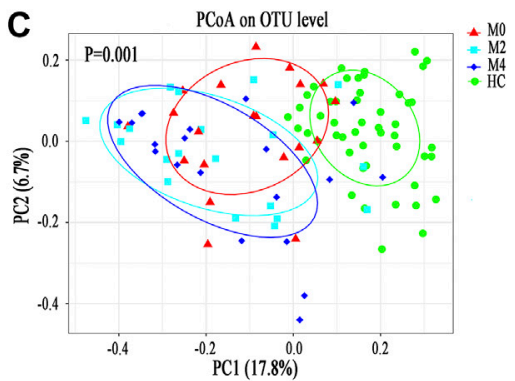

G

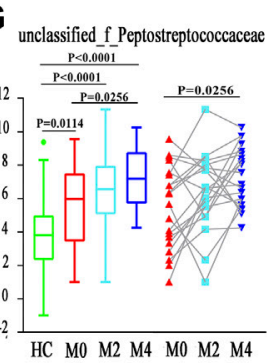

H

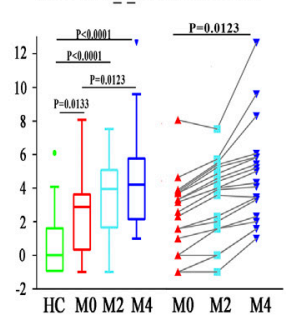

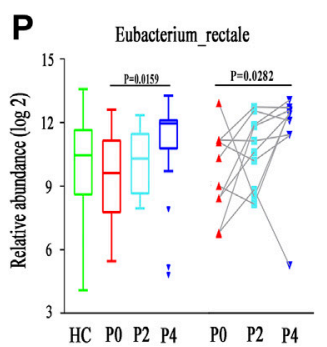
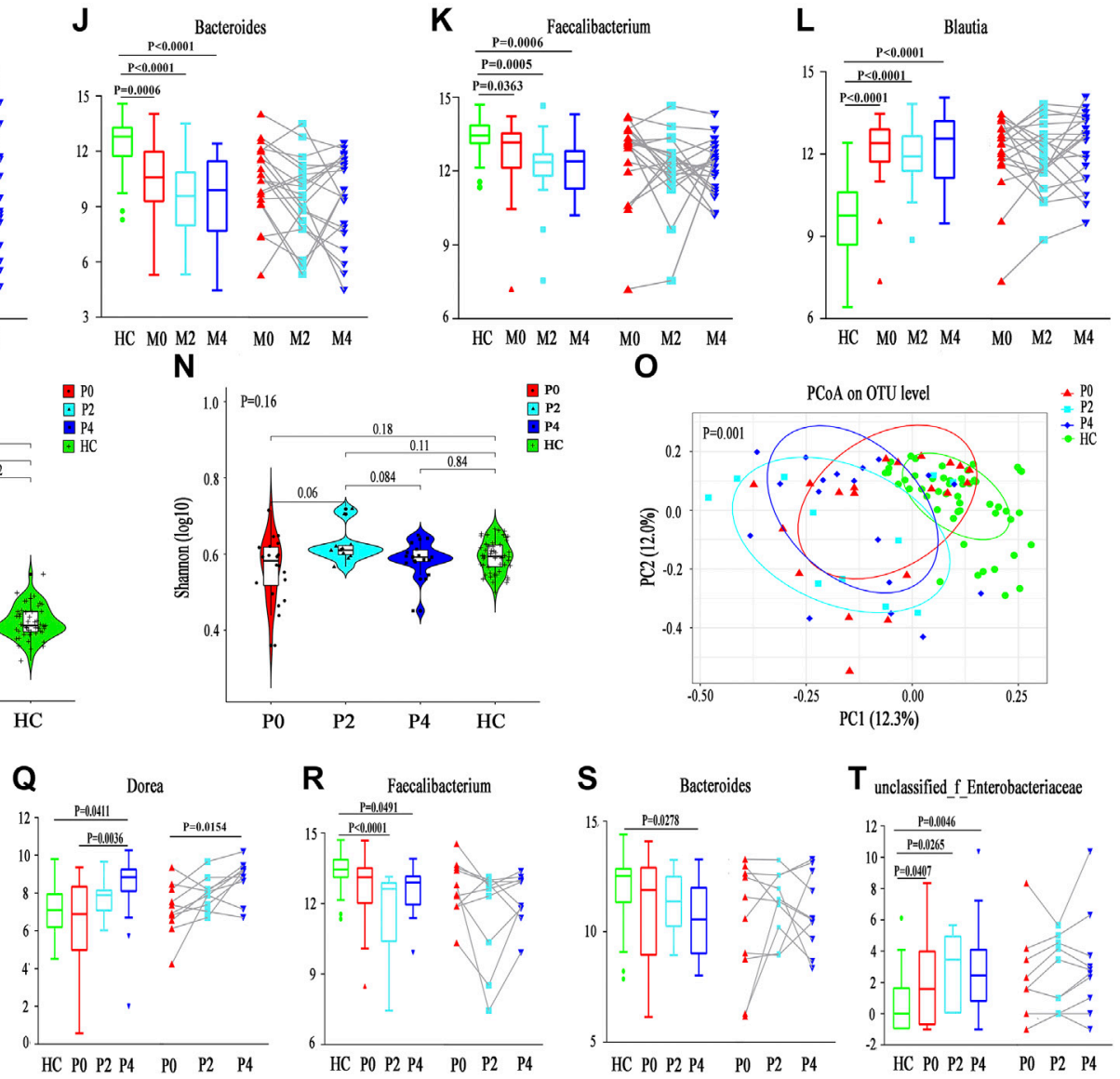

FIGURE 2 | Longitudinal changes in the gut microbiota diversity and structure between MMI and PTU. (A,B) Violin plot of the alpha diversity indexes for richness (Ace index) and evenness (Shannon index) of the gut microbiota among the M0, M2, M4, and HC groups. (C) PCoA analysis based on the Bray-Curtis distance showing different clusters among the four groups $(p=0.001)$. (D-L) Unpaired test (left) and paired test (right) comparisons of the relative abundances in each pair of the M0, M2, M4, and HC groups. (M,N) Violin plot of the alpha diversity Ace index and Shannon index of the gut microbiota among the P0, P2, P4, and HC groups. (O) PCoA based on the Bray-Curtis distance showing different clusters among the four groups $(p=0.001)$. (P-T) Unpaired t test (left) and paired t test (right) comparisons of the relative abundances in each pair of the $\mathrm{PO}, \mathrm{P} 2, \mathrm{P} 4$, and $\mathrm{HC}$ groups. 
Supplementary Figure S4G). However, an additional 28 discriminative features, representing one phylum, 10 families and 17 genera, were observed among low-abundance microbiota constituents (Supplementary Figures S4H-J). Similar to in the MMI group, Faecalibacterium, Bacteroides and unclassified_f_Enterobacteriaceae, had no statistical significance in comparisons among the three time points (P0 vs. P2 vs. P4) but were significantly different from $\mathrm{HCs}$ and showed longitudinal changes are shown in Figures 2R-T; and the low-abundance discriminative microbiota explained separation result of the PCoA.

In summary, the longitudinal changes in the gut microbiota structure in the MMI and PTU groups reflect not only the effect of ATDs on the microbiota but also the effects of different ATDs; for example, Romboutsia abundance increased mainly in the MMI group, while Eubacterium_rectale and Dorea abundances increased mainly in the PTU group. In addition, microbiota dysbiosis developed in both the Init_GD and Treat_GD groups, and the dysbiosis was aggravated at the 2 nd week and showed partial recovery by the 4 th week.

\section{Associations Between the Gut Microbiota and Clinical Indexes}

To explore the effect of changes in the gut microbiota structure on phenotypes, we further investigated the correlation between the relative abundance of all differentially abundant genera $(n=47)$ and clinical parameters $(n=38)$ using Spearman's correlation analysis (Supplementary Figure S5). The abundance levels of Treat_GD group-enriched genera, including Blautia, Streptococcus and Dorea, were positively correlated with some liver function-related indicators, such as ALT, AST and AKP; however, these indicators were negatively correlated with some HC-enriched genera, including Faecalibacterium and Bacteroides (Figure 3A). These microbiota constituents all participate in liver function regulation, which may also be correlated with ATDs-induced liver injury (Chen et al., 2014; Shen et al., 2017; Meng et al., 2018). In addition, the clinical parameters fT3, fT4, Tg-Ab, and TPO-Ab were positively correlated with Lactobacillus, Streptococcus, Blautia, etc., and negatively correlated with Roseburia, Dialister, Alistipes, etc., while TSH showed opposite results. Moreover, the changes in LPS-producing microbiota constituents, such as Klebsiella and Escherichia-Shigella, were positively correlated with the LPS variations in the fecal supernatant and serum (Figure 3A). Klebsiella abundance was also positively correlated with liver function indexes, such as ALT, AST and AKP.

\section{Functional Alterations in the Gut Microbiota}

As mentioned above, both the gut microbiota and clinical parameters were altered after ATDs treatment and showed a certain correlation. The role that the gut microbiota plays is unknown, and thus, Phylogenetic Investigation of Communities by Reconstruction of Unobserved States (PICRUSt) of all gut microbiota, which can predict the function of gut microbiota, was performed. We hypothesized that the disrupted gut microbiota plays key roles in ATDs-induced liver injury through the intestinal endotoxemia hypothesis, and the mainly related four metabolic pathways were analyzed.

Lipopolysaccharide biosynthesis $(p<0.0001)$ and Lipopolysaccharide biosynthesis protein $(p<0.0001)$ pathways were more enriched in the Init_GD and Treat_GD groups than in the HC group, which consistent with LPS variations in the fecal supernatant and serum (Figures 3B,C). In addition, Bacterial invasion of epithelial cells and Bacterial toxins pathways, which are associated with bacterial translocation from the intestine, impaired metabolic homeostasis and, subsequently, chronic low-grade inflammation, were also evaluated and were more enriched in the Init_GD and Treat_GD groups than in the HC group (Figure 3C). Similarly, PICRUSt was performed among different ATDs and different time points. All four pathways showed higher activity in the MMI and PTU groups than in the $\mathrm{HC}$ group, although there were no significant differences among them (Figure 3D). The four pathways showed higher activation in the Init_GD group, and they exhibited generally increasing trends with MMI or PTU use (Figures 3E,F).

In summary, consistent with the change in the gut microbiota structure, the gut microbiota function may changed along with the change of gut microbiota structure, and these changes were correlated with clinically relevant phenotypes. In particular, the increased activity of pathways associated with the intestinal endotoxemia hypothesis is consistent with the change in LPS concentration in the fecal supernatant and serum, which serves as a preliminary clinical validation of our hypothesis that the gut microbiota participates in ATDs-induced side effects through the intestinal endotoxemia hypothesis.

\section{Part 2: Animal Study}

The animal studies not only repeated the above clinical study but also provided another intervention (i.e., different doses) and displayed related organizational structural changes.

\section{Study Subjects and Baseline Indicators}

Female age- and weight-matched (6-8 weeks old, weighing $200 \pm 10 \mathrm{~g})$ SD rats were purchased from the animal experiment center of the Second Affiliated Hospital of Harbin Medical University and raised in the same environment. Thyroid function decreased gradually with the use of ATDs. At the 4th week, fT3 and fT4 levels showed no significant differences when compared with baseline, while the TSH level showed differences among subgroups, such as between the $\mathrm{MH}$ and $\mathrm{PL}$ groups. At the 6th week, fT3 and fT4 levels also showed differences from the baseline in some subgroups, indicating the occurrence of hypothyroidism, and fT3, fT4, and TSH levels all showed significant differences from the baseline at the 10th week in all groups (Table 2). Consistent with the serum results, the rats in the ATDs group exhibited rough hair, reduced food intake and relatively slow movement with the development of hypothyroidism. The rat body weight in the Control one group increased, while the rat body weight in the ATDs group decreased, and the PTU group decreased more (Table 2). However, it is worth noting that body weight is usually increased in rats with hypothyroidism (Kyriacou et al., 2019); this conflicting result 


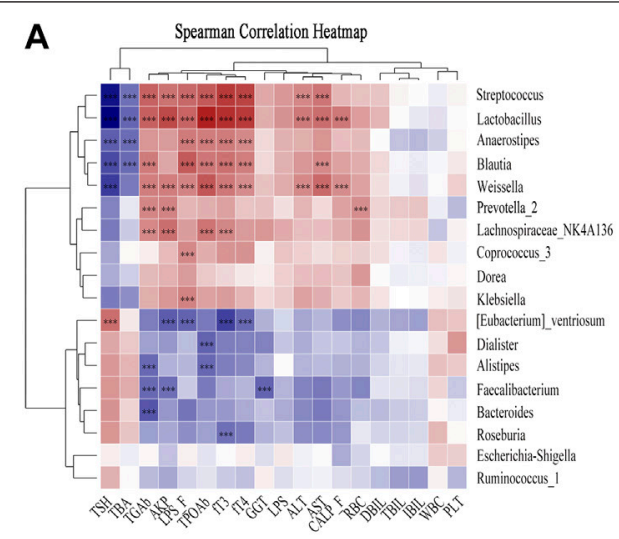

B
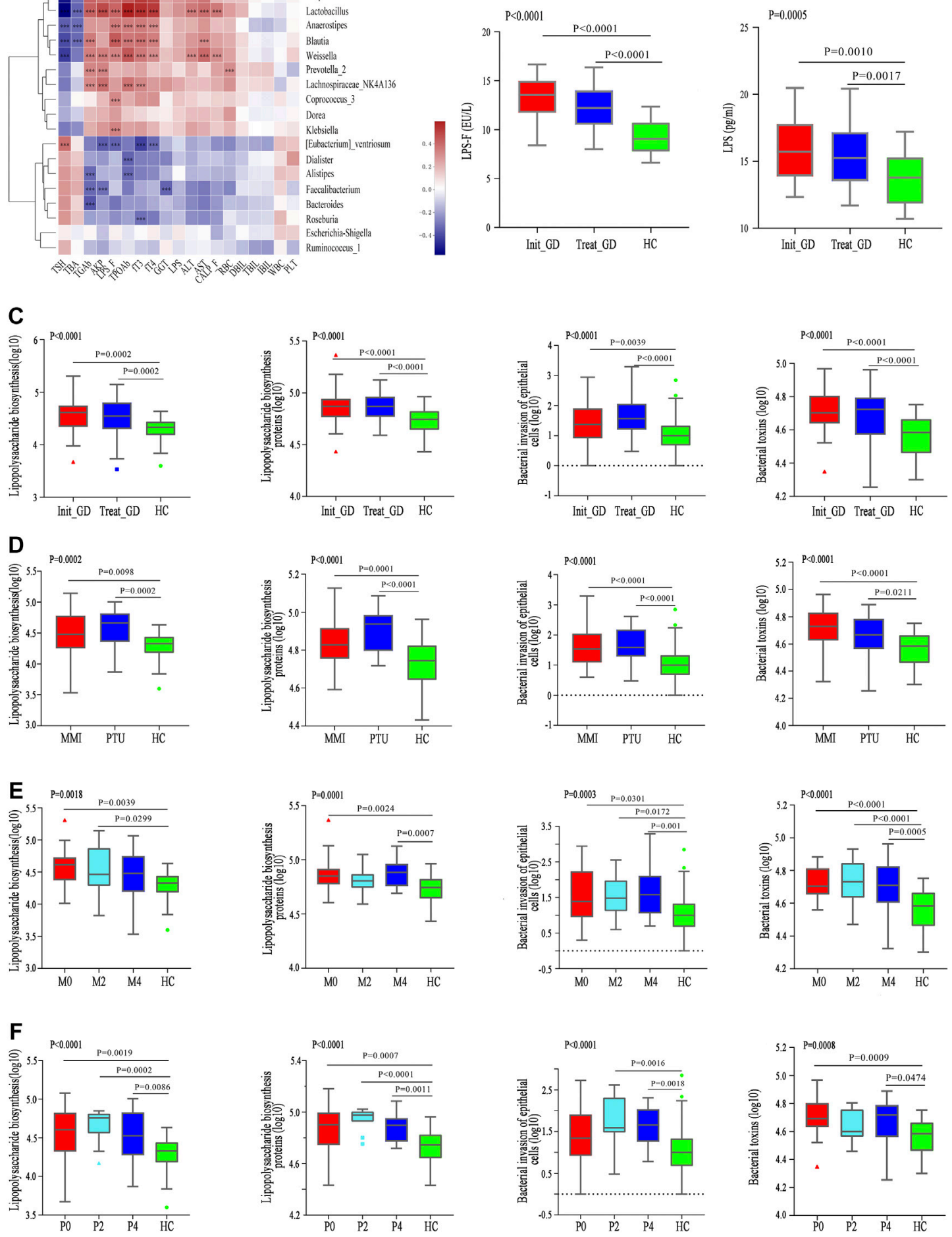

FIGURE 3 | Correlation between differential microbiota and basal indicators, and change of KEGG and gut barrier related indexes. (A) Spearman correlation analysis of 19 clinical indicators and 18 differential genera; color intensity represents the magnitude of correlation. Red, positive correlations; blue, negative correlations. ${ }^{\star} p$ value $<0.05 ;{ }^{\star \star} p$ value $<0.01$; ${ }^{\star \star \star} p$ value $<0.001$. (B) LPS concentration in serum (LPS) and fecal supernatant (LPS_F). (C-F) The change of pathway Lipopolysaccharide biosynthesis, Lipopolysaccharide biosynthesis proteins, Bacterial invasion of epithelial cells, and Bacterial toxins among groups with ATDs, different ATDs and different time points. 
TABLE 2 | Basic parameters of SD rats.

\begin{tabular}{|c|c|c|c|c|c|}
\hline & Group & Baseline & 4th week & 6th week & 10th week \\
\hline \multirow[t]{5}{*}{ fT3 } & $\mathrm{ML}$ & $6.09 \pm 0.75$ & $6.39 \pm 0.54$ & $5.61 \pm 0.85$ & $3.8 \pm 0.59^{\star \star \star}$ \\
\hline & $\mathrm{MH}$ & $5.76 \pm 0.59$ & $5.66 \pm 0.46$ & $4.56 \pm 0.32^{\star \star}$ & $3.82 \pm 0.62^{\star \star \star}$ \\
\hline & $\mathrm{PL}$ & $6.84 \pm 0.63$ & $6.14 \pm 0.71$ & $5.97 \pm 0.63^{\star}$ & $4.88 \pm 0.49^{\star \star \star}$ \\
\hline & $\mathrm{PH}$ & $5.42 \pm 0.45$ & $6 \pm 0.87$ & $5.21 \pm 0.48$ & $4.43 \pm 0.7^{\star \star}$ \\
\hline & ATDs & $6.06 \pm 0.8$ & $6.05 \pm 0.69$ & $5.43 \pm 0.78^{\star \star}$ & $4.35 \pm 0.73^{\star \star \star}$ \\
\hline \multirow[t]{5}{*}{ fT4 } & $\mathrm{ML}$ & $19.9 \pm 2.03$ & $19.47 \pm 2.69$ & $17.68 \pm 1.67^{\star}$ & $14.87 \pm 2.49^{\star \star}$ \\
\hline & $\mathrm{MH}$ & $20.97 \pm 3.06$ & $18.53 \pm 1.81$ & $13.49 \pm 2.32^{\star \star}$ & $13.61 \pm 1.34^{\star \star \star}$ \\
\hline & $\mathrm{PL}$ & $21.42 \pm 2.06$ & $19.76 \pm 2.26$ & $19.7 \pm 2.86$ & $16.15 \pm 2.37^{\star \star \star}$ \\
\hline & $\mathrm{PH}$ & $19.73 \pm 3.03$ & $20.81 \pm 2.23$ & $17.14 \pm 2.48$ & $14.48 \pm 3.02^{\star \star}$ \\
\hline & ATDs & $20.53 \pm 2.54$ & $19.68 \pm 2.3$ & $17.38 \pm 3.07^{\star \star \star}$ & $14.92 \pm 2.48^{\star \star \star}$ \\
\hline \multirow[t]{5}{*}{ TSH } & $\mathrm{ML}$ & $11.68 \pm 2.18$ & $12.72 \pm 1.28$ & $13.4 \pm 1.71$ & $15.01 \pm 2.17^{\star}$ \\
\hline & $\mathrm{MH}$ & $11.62 \pm 0.92$ & $13.33 \pm 1.85^{\star}$ & $13.69 \pm 1.56^{\star}$ & $16.77 \pm 2.93^{\star \star}$ \\
\hline & $\mathrm{PL}$ & $9.66 \pm 1.8$ & $12.06 \pm 1.97^{\star}$ & $10.03 \pm 1.06$ & $12.39 \pm 2.16^{\star}$ \\
\hline & $\mathrm{PH}$ & $11.29 \pm 1.41$ & $10.9 \pm 1.48$ & $14.1 \pm 1.64^{\star \star}$ & $14.15 \pm 0.92^{\star \star \star}$ \\
\hline & ATDs & $11.01 \pm 1.78$ & $12.22 \pm 1.83^{\star}$ & $12.66 \pm 2.23^{\star \star}$ & $14.25 \pm 2.54^{\star \star \star}$ \\
\hline \multirow[t]{5}{*}{ Weight } & $\mathrm{ML}$ & $237.67 \pm 10.87$ & $244.44 \pm 16.44$ & $232.98 \pm 16.95$ & $238.73 \pm 7.8$ \\
\hline & $\mathrm{MH}$ & $228.51 \pm 15.07$ & $230.11 \pm 21.67$ & $227.62 \pm 25.31$ & $219.68 \pm 15.93$ \\
\hline & $P L$ & $230.79 \pm 11.66$ & $224.58 \pm 18.48$ & $210.3 \pm 20.29^{\star}$ & $199.9 \pm 13.46^{\star \star \star}$ \\
\hline & $\mathrm{PH}$ & $244.77 \pm 8.74$ & $223.46 \pm 11.67^{\star \star}$ & $205.31 \pm 15.27^{\star \star \star}$ & $194.83 \pm 15.18^{\star \star \star}$ \\
\hline & Control 1 & $248.6 \pm 12.38$ & $280.88 \pm 15.5^{\star}$ & $287.28 \pm 9.6^{\star \star}$ & $319.25 \pm 8.84^{\star \star \star}$ \\
\hline
\end{tabular}

The Wilcoxon rank-sum test was used to compare these parameters and values are expressed as the mean $\pm S D$. Statistical difference from corresponding baseline expressed as $\mathrm{p}$ values: ${ }^{*} \mathrm{p}<0.05,{ }^{* *} \mathrm{p}<0.01,{ }^{* * *} \mathrm{p}<0.001$.

may be because of the reduced food intake and malnutrition after ATDs administration, which is consistent with the lower ALB level in the ATDs group than in the control group (Supplementary Table S4). The changes in the liver, spleen, thyroid and gastrointestinal weight are shown in Supplementary Figure S6A. Interestingly, after group matching and adjusting for weight, we found that the gastrointestinal tissue weight was significantly correlated with body weight. In contrast, the thyroid weight was higher in the ATDs group than in the control group, while the liver and spleen weights were lower in the ATDs group. In the high-dose group, both MMI- and PTUadministered rats showed obvious trends though the liver weight was greater in the high-dose group than in the low-dose group. The increase in thyroid weight may be due to reactive hyperplasia, which is consistent with the enlarged volume of the thyroid at the 2ndweek compared with that in the control group (Supplementary Figure S6B) (Shibutani et al., 2009). The decrease in liver and spleen weight may be due to ATDsinduced injury, which is consistent with the results for the following biochemical indicators (Cano-Europa et al., 2011; Fukui et al., 2013).

In the animal study, liver function-related indexes, such as ALT, GGT, TBIL, DBIL and total bile acid (TBA), increased in both ATDs groups (the MMI and PTU groups) compared with those in the control group, while nutrition-related indicators, such as ALB, decreased (Supplementary Table S4). In addition, the ALT level increased more in the PTU group than in the MMI group ( $p=0.033$ ), while the TBA increased more in the MMI group $(p=0.011)$, which is consistent with the findings that MMI always causes cholestatic liver injury and that PTU often causes hepatocyte liver injury (Wang et al., 2014).

\section{Gut Microbiome Profiles Among Groups Sequencing Characteristics}

From all 123 fecal samples, 9,249,423 high-quality sequences were selected based on barcode- and primer-sequence filtering, with 75,199 (ranging from 51,739 to 98,762) sequences per barcoded sample. A total of 3,338 OTUs were delineated at a $97 \%$ similarity level. The rarefaction curve of all samples reached a plateau (Supplementary Figure S6C), and the average Good's coverage for these samples was greater than 99.3\%, which indicated a satisfactory gut microbiota sequencing depth. Furthermore, we divided these samples into 24 subgroups according to the aims of the study. The specific information is shown in Supplementary Table S5. The aims were as follows:

\section{Part 1: To Further Investigate Whether Antithyroid Drugs Can Cause Changes in the Gut Microbiota Structure (Antithyroid Drugs Group vs. Control Group) Alpha and Beta Diversity}

The alpha diversity of the gut microbiota in the ATDs group ( $n=82$, including all the samples after ATDs administration) was significantly higher than that in the control group $(n=41$, including all the samples before ATDs administration and the first three time points of Control one group) according to the Ace index $(p=5.1 \mathrm{e}-15)$, the Shannon index $(p=5.7 \mathrm{e}-13)$ and other diversity indexes (Figures 4A,B; Supplementary Table S5); the increased Ace and Shannon indexes after ATDs administration were consistent with the tendency for clinical variation, although no significant differences were found in the clinical study. PCoA revealed significant separation of the gut microbiota structure between the ATDs group and the control group $(p=0.001$, Figure 4C), revealing a more obvious distinction than that in the 


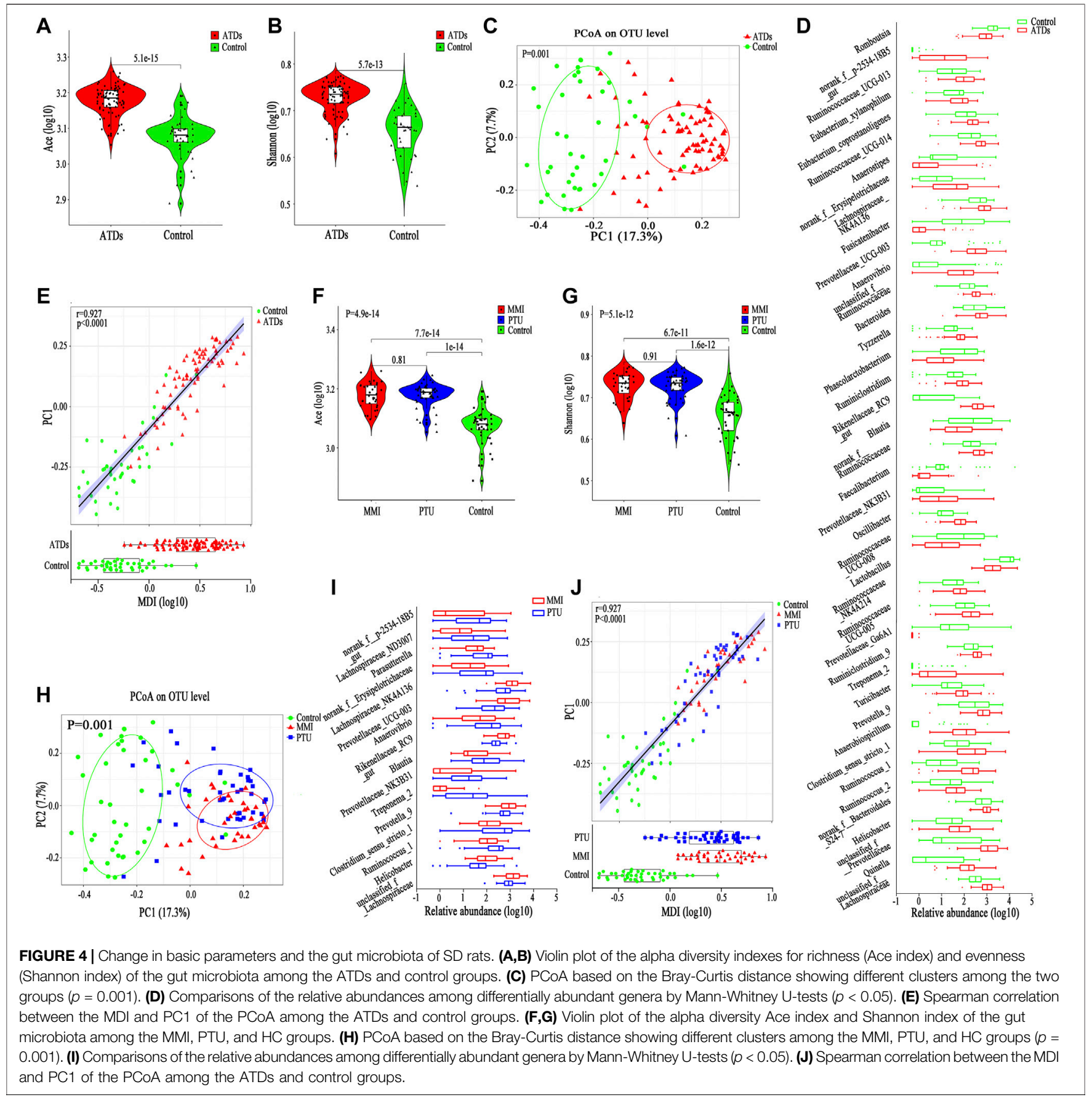

clinical comparison of the Treat_GD and Init_GD groups. To show the specific changes caused by ATDs, we performed a differentially abundant species analysis.

\section{Differentially Abundant Species Analysis}

We compared the gut microbiota compositions between the ATDs and control groups, and 60 differentially abundant species, representing four phyla, 15 families and 41 genera. At the phylum level, Bacteroidetes, Proteobacteria and Spirochaetae abundances were enriched in the ATDs group, whereas Firmicutes abundance was enriched in the control group (Supplementary Figure S6D). Interestingly, the Firmicutes proportion was increased, while the Bacteroidetes proportion was decreased in the clinical study, which may be due to the different microbiotas between humans and rats, to the different disease statuses, or to the fact that clinical cases are often accompanied by adjuvant drug interference. At the family level, the ATDs group-enriched species included mainly Prevotellaceae 
and Ruminococcaceae, while Lactobacillaceae and Peptostreptococcaceae were prevalent in the control group (Supplementary Figure S6D). Similar to at the phylum level, the shared differentially abundant families in humans and animals, Ruminococcaceae and Peptostreptococcaceae, showed different variation trends. Lactobacillus, Romboutsia and Faecalibacterium were enriched in the control group, while Clostridium_sensu_stricto_1, Prevotellaceae_UCG-003 and Oscillibacter were enriched in the ATDs group (Figure 4D). The MDI also increased after ATDs intervention compared with that in the controls in the animal study, which was significantly correlated with PC1 ( $r=$ 0.927, $p<0.0001$, Figure 4E), reflecting that the gut microbiota structure was disturbed in the ATDs group.

Part 2: To Further Investigate Whether Different Antithyroid Drugs Cause Different Gut Microbiota Structure Changes (Methimazole vs. Propylthiouracil vs. Control; MMI_2 Vs. PTU_2, Including Subgroups With Specific Doses; MMI_3 Vs. PTU_3, Including Subgroups With Specific Doses; MMI_4 vs. PTU_4, Including Subgroups With Specific Doses)

\section{Alpha and Beta Diversity}

Both the MMI and PTU groups showed higher Ace and Shannon indexes than the control group, but there was no significant difference between the two drug groups (Figures $4 \mathrm{~F}, \mathbf{G}$ ). Interestingly, before the occurrence of hypothyroidism (at the 4 th week and/or the 6th week), the Ace and Shannon indexes of the MMI group were higher than those of the PTU group, although they showed no significant difference, which is consistent with the changes observed in the clinical study after ATDs administration (Supplementary Table S5).

PCoA showed that both the MMI and PTU groups deviated from the control group. The MMI group was farther from the control group than the PTU group ( $p=0.001$, Figure $4 \mathbf{H}$ ), which can also be seen from the separate comparison between MMI and PTU groups $(p=0.001$, Supplementary Figure S6E). Interestingly, PCoA showed that different subgroups clustered slowly following the administration of ATDs, which may be because hypothyroidism aggravation plays a dominant role in the gut microbiota structure. On the other hand, before the occurrence of hypothyroidism (at the 4th week and/or the 6th week), subgroups within the MMI and PTU groups exhibited relatively greater separation from one another, reflecting the different effects caused by different ATDs on the gut microbiota (Supplementary Figures S6F-H).

\section{Differentially Abundant Species Analysis}

To identify the specific structural changes associated with the administration of different ATDs, we compared the gut microbiota compositions in the MMI and PTU groups in the animal study, and 24 discriminative species, representing one phylum, seven families (Supplementary Figure S7A) and 16 genera (Figure 4I), were revealed with $p<0.05$. At the phylum level, Spirochaetae was more prevalent in the PTU group than in the MMI group; the corresponding families Spirochaetaceae and Clostridiaceae_1 also showed higher abundance in the PTU group, while Lachnospiraceae and Rikenellaceae were more prevalent in the MMI group (Supplementary Figure S7A). At the genus level, Prevotella_9 and Rikenellaceae_RC9 were enriched in the MMI group, while Clostridium_sensu_stricto_1, Blautia, and Treponema_2 were more prevalent in the PTU group (Figure 4I). Although the microbiota in humans and rats differ and there were a few shared differentially abundant microbiota constituents, the MMI and PTU groups showed differentially abundant microbiota constituents in both the clinical and animal studies.

Similarly, the MDI significantly increased in both the MMI and PTU groups compared with that in the control group, and the MMI group exhibited a higher MDI than the PTU group, which is consistent with the clinical study results. Furthermore, the MDI was significantly correlated with PC1 $(r=0.927, p<0.0001$, Figure 4J), illustrating that gut microbiota dysbiosis occurred in both the MMI and PTU groups, while the MMI group showed more dysbiosis.

\section{Part 3: To Investigate Whether the Antithyroid Drugs-Induced Changes in the Gut Microbiota Structure Are Dose Dependent (ML_234 vs. MH_234; ML_2 vs. MH_2; ML_3 vs. MH_3; ML_4 vs. MH_4; PL_234 vs. PH_234; PL_2 vs. $\mathrm{PH} \_2 ; \mathrm{PL}$ 3 vs. $\mathrm{PH}$-3; and $\mathrm{PL}_{4} 4$ vs. $\mathrm{PH} 4$ ) \\ Alpha and Beta Diversity}

Interestingly, in the MMI group, the alpha diversity index after ATDs administration was higher in the ML_234 group than in the MH_234 group, although there were no significant difference (Ace, $p=0.68$; Shannon, $p=0.33$, Figures 5A,B). In the PTU group, neither the Ace index $(p=0.62)$ nor the Shannon index $(p=0.87)$ showed a significant difference between the PL_234 and PH_234 groups, while the different indexes showed different variation trends (Figures 5C,D). For both the MMI and PTU groups, PCoA showed that subgroups corresponding to different doses deviated from one another (Figures 5E-H). Similar to the different ATD groups, the subgroups corresponding to different doses exhibited greater separation at the 4 th and 6 th weeks and had a tendency to cluster together as the ATDs intervention progressed (Supplementary Figures S7B-D). By the 10 th week, there was no significant difference between the ML and MH groups ( $p=0.081$, Supplementary Figure S7D), which may be due to the dominant role of hypothyroidism.

\section{Differentially Abundant Species Analysis}

In the MMI group, 17 discriminative species, representing two phyla, five families ( $p<0.05$, Supplementary Figure S7E) and 10 genera $(p<0.05$, Figure 5I), were found between the ML and MH groups. Consistent with the alpha diversity result, the abundance of the most discriminative microbiota constituent was relatively higher in the ML group than in the $\mathrm{MH}$ group. For example, at the phylum level, both the differentially abundant phyla Proteobacteria and Spirochaetae were prevalent in the ML group. Similarly, at the family level, a higher abundance of Desulfovibrionaceae and Christensenellaceae was found in the ML group, while Streptococcaceae was more prevalent in the $\mathrm{MH}$ 


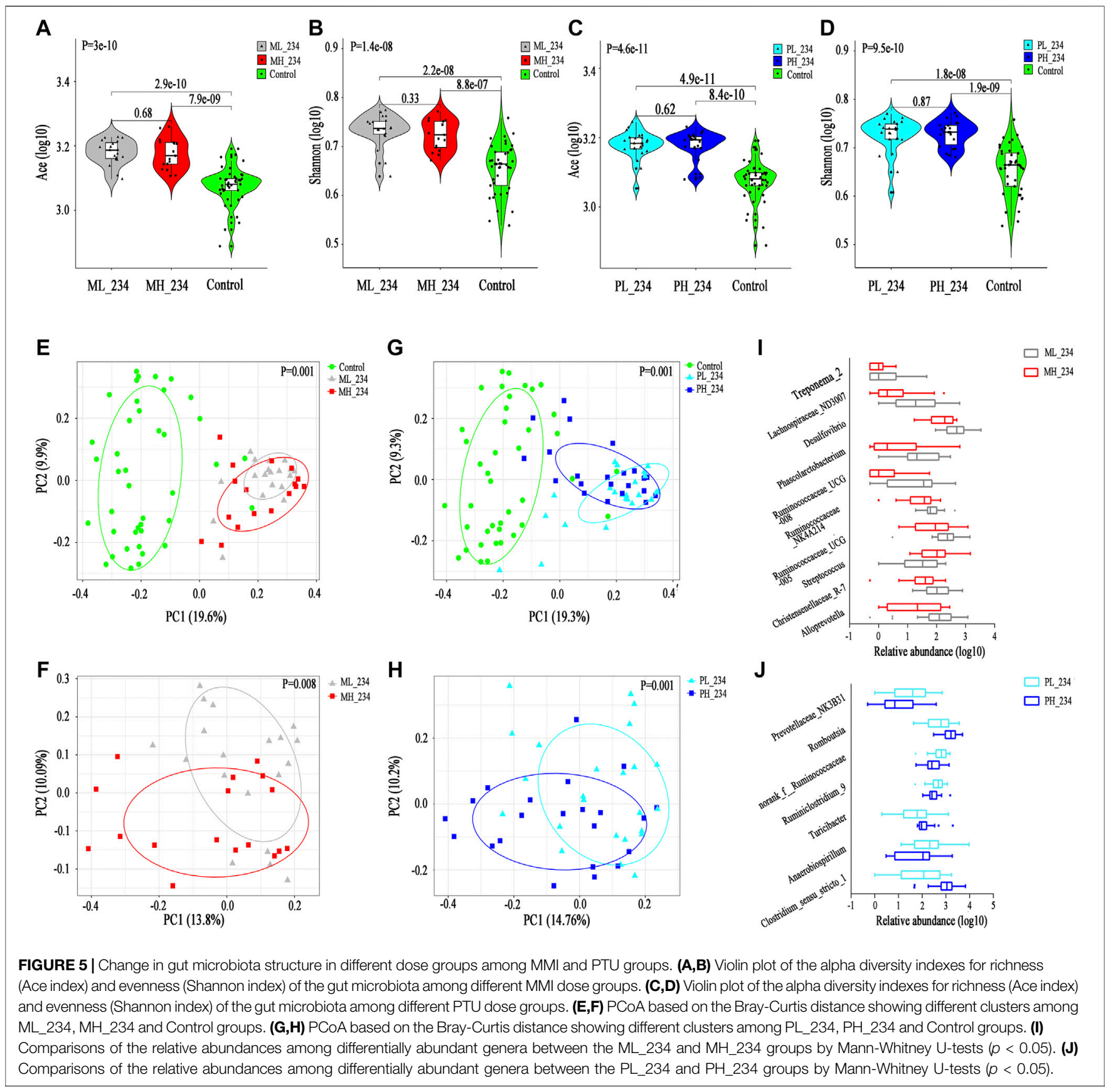

group, and the corresponding genera Desulfovibrio and Streptococcus showed the same trend. In the PTU group, ten discriminative features, representing three families $(p<0.05$, Supplementary Figure S7F) and seven genera $(p<0.05$, Figure 5J), were revealed between the PL and PH groups. At the family level, Peptostreptococcaceae and Clostridiaceae_1 were abundant in the $\mathrm{PH}$ group, and Succinivibrionaceae was abundant in the PL group. The genera Romboutsia and Clostridium_sensu_stricto_1 showed a higher abundance in the PH group than in the PL group, while Anaerobiospirillum showed a lower abundance.
Part 4: To Further Investigate the Longitudinal Antithyroid Drugs-Induced Changes in the Gut Microbiota Structure at Different Time Points (ML_1 vs. ML_2 vs. ML_3 vs. ML_4; MH_1 vs. MH_2 vs. MH_3 vs. $M H_{-} 4$; $P L \_1$ vs. $P L \_2$ vs. $P L \_3$ vs. $P L \_4 ; P H \_1$ vs. $\mathrm{PH} \_2$ vs. $\mathrm{PH} \_3$ vs. $\mathrm{PH} \_4$; all Include Comparisons Between One Another)

We next explored the longitudinal changes in the gut microbiota structure in four cohorts (the ML group, MH group, PL group and $\mathrm{PH}$ group) to eliminate the interference of different doses and drugs. 


\section{Alpha and Beta Diversity}

Both the Ace and Shannon index gradually increased and then tended to stabilize along with ATDs application in all four cohorts (Figures 6A-H; Supplementary Table S5). Moreover, the PCoA results showed different clusters in the gut microbiota structure distribution at different time points among the four cohorts (Figures 6I-L); however, the distances between distributions decreased, which may be due to hypothyroidism progression.

\section{Differentially Abundant Species Analysis}

Next, we performed a longitudinal analysis of differentially abundant microbiota constituents among the four cohorts, including a comprehensive analysis of the four time points and comparisons of each pair of time points. In the ML group, 63 discriminative species, representing four phyla, 17 families $(p<0.05$, Supplementary Figure S8A) and 42 genera $(p<0.05$, Supplementary Figure S9A), were revealed; in the MH group, 50 discriminative species, representing two phyla, 13 families $(p<0.05$, Supplementary Figure S8B) and 35 genera $(p<0.05$, Supplementary Figure S9B), were revealed; in the PL group, 56 discriminative species, representing four phyla, 17 families $(p<0.05$, Supplementary Figure S8C) and 35 genera $(p<0.05$, Supplementary Figure S9C), were revealed; and in the $\mathrm{PH}$ group, 57 discriminative species, representing five phyla, 16 families $(p<0.05$, Supplementary Figure S8D) and 36 genera, were revealed $(p<0.05$, Supplementary Figure S9D).

With ATDs administration, most microbiota constituents showed a longitudinal gradient change. At the phylum level, Firmicutes abundance significantly decreased and Bacteroidetes abundance increased in all cohorts except for the MH group, which showed the opposite trend, although there was no significant difference in the $\mathrm{MH}$ and $\mathrm{PH}$ groups. At the family level, Lactobacillaceae abundance decreased and Ruminococcaceae abundance increased with ATDs administration. Similarly, at the genus level, some genera varied in both the MMI and PTU groups, such as Lactobacillus, which decreased in abundance with ATDs use, while unclassified_f_Prevotellaceae abundance increased. However, some microbiota constituents showed different trends among different ATD treatments. For example, Alloprevotella abundance increased in the MMI group and decreased in the PTU group, although it varied in both drug administration groups. Romboutsia and Lachnospiraceae_NK4A136 abundances varied mainly in the MMI group, while Desulfovibrio abundance varied mainly in the PTU group, which also reflects the different effects of different ATDs on the gut microbiota structure.

\section{Associations Between the Gut Microbiota and Basal Indicators}

The gut microbiota structure also changed after ATDs administration (including use or nonuse of drugs, different drugs, different doses and different time points). The correlation between the relative abundance of all differentially abundant genera $(n=49)$ and basal indicators $(n=21)$ was also analyzed by Spearman's correlation in the animal study
(Supplementary Figure S10). The abundances of ATDs group-enriched genera, such as Prevotellaceae_UCG-003, Oscillibacter and Rikenellaceae_RC9, were positively correlated with liver function-related indicators, such as GGT, TBIL, DBIL and TBA. However, these indicators were negatively correlated with the abundances of some control group-enriched genera, such as Lactobacillus, Prevotellaceae_Ga6A1 and Faecalibacterium (Figure 7A). The correlation analysis showed that the change in the gut microbiota structure induced by ATDs may be correlated with liver function injury. Importantly, the microbiota constituents that were positively correlated with the liver function indexes were also positively correlated with LPS content in the fecal supernatant and serum (Figure 7A), and some genera, such as Oscillibacter, were enriched in an animal model of nonalcoholic fatty liver disease and positively correlated with plasma LPS content and intestinal permeability. Other clinical parameters, such as fT3 and fT4 levels, were positively correlated with Lactobacillus, Fusicatenibacter and Faecalibacterium and negatively correlated with Oscillibacter, Prevotellaceae_UCG-003, Rikenellaceae_RC9, etc. The TSH level showed an opposite relation with these microbiota constituents (Figure 7A).

\section{Functional Alterations in the Gut Microbiota}

To characterize the functional alterations in the gut microbiota in the animal study, we also predicted the functional composition profiles using $16 \mathrm{~S}$ rRNA sequencing data analyzed with PICRUSt. Similar to those in the clinical results, the metabolic pathways that were analyzed involved mainly pathways related to intestinal endotoxemia hypothesis.

Similar to the significant changes in the gut microbiota structure, the pathways of the Lipopolysaccharide biosynthesis, Lipopolysaccharide biosynthesis proteins, Bacterial invasion of epithelial cells, and Bacterial toxins were significantly enriched in the ATDs group compared with those in the control group (Figure 7C). Similarly, both the MMI and PTU groups showed increases in these four pathways compared with those in the control group, although no significant differences were found between the MMI and PTU groups. The Lipopolysaccharide biosynthesis and Lipopolysaccharide biosynthesis protein pathways were enhanced in the MMI group, which was consistent with the elevated LPS concentration in the fecal supernatant and serum in the MMI group. However, the Bacterial invasion of epithelial cells and Bacterial toxin pathways were higher in the PTU group than in the MMI group (Figure 7D). In the different dose groups, the four pathways were more enriched in the high-dose group than in the low-dose group, although most of them showed no significant difference (Figures 7E,F). Finally, the longitudinal changes among specific doses in the MMI and PTU groups were analyzed and showed an overall increasing trend following ATDs administration, which was also consistent with the changes in LPS content in the fecal supernatant and serum (Figures 7B,G-J).

The dysbiosis of the gut microbiota structure and function, accompanied by the increasing LPS concentration and corresponding changes in basal indicators, support our hypothesis. Gut microbiota composition alterations may be 


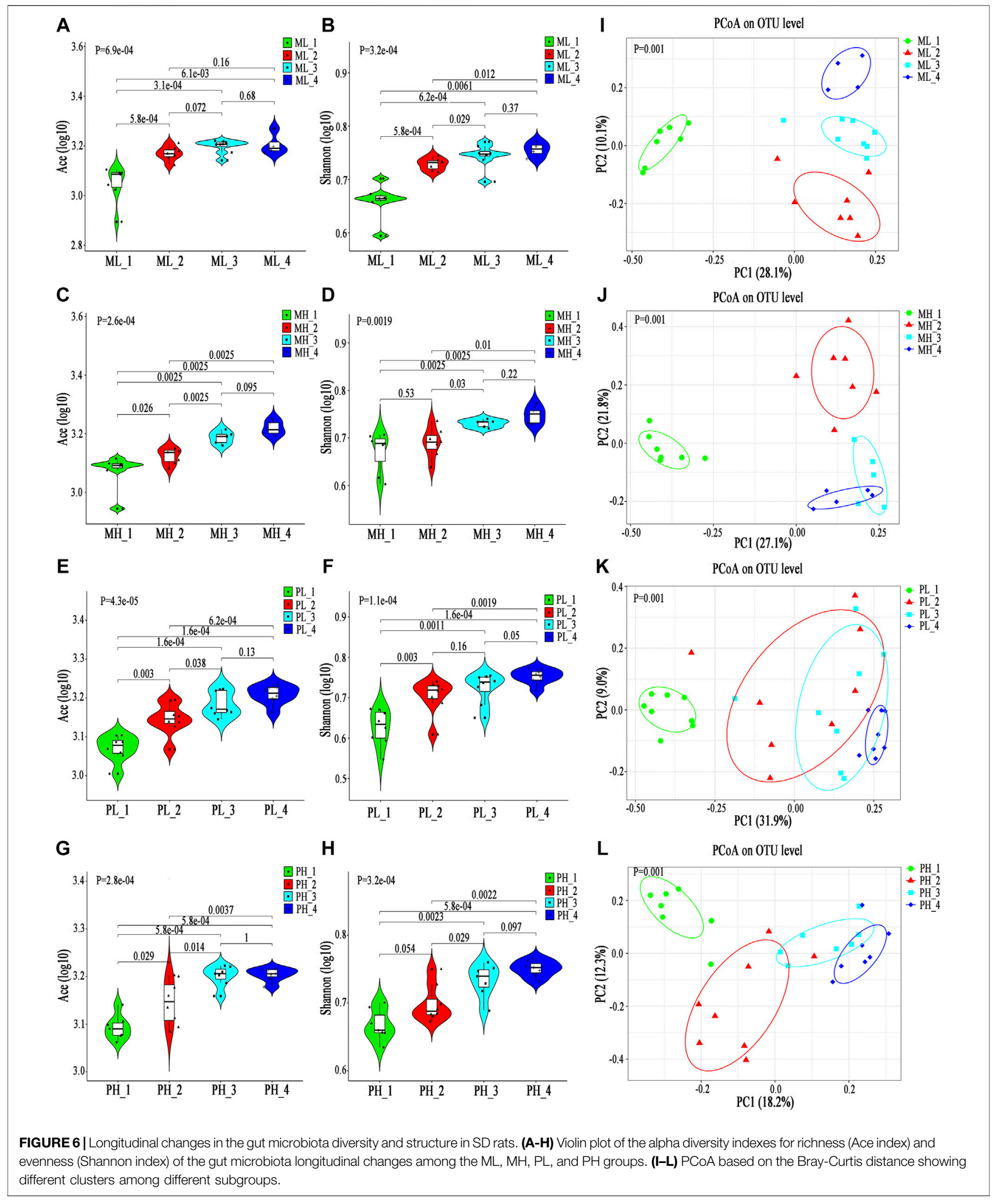



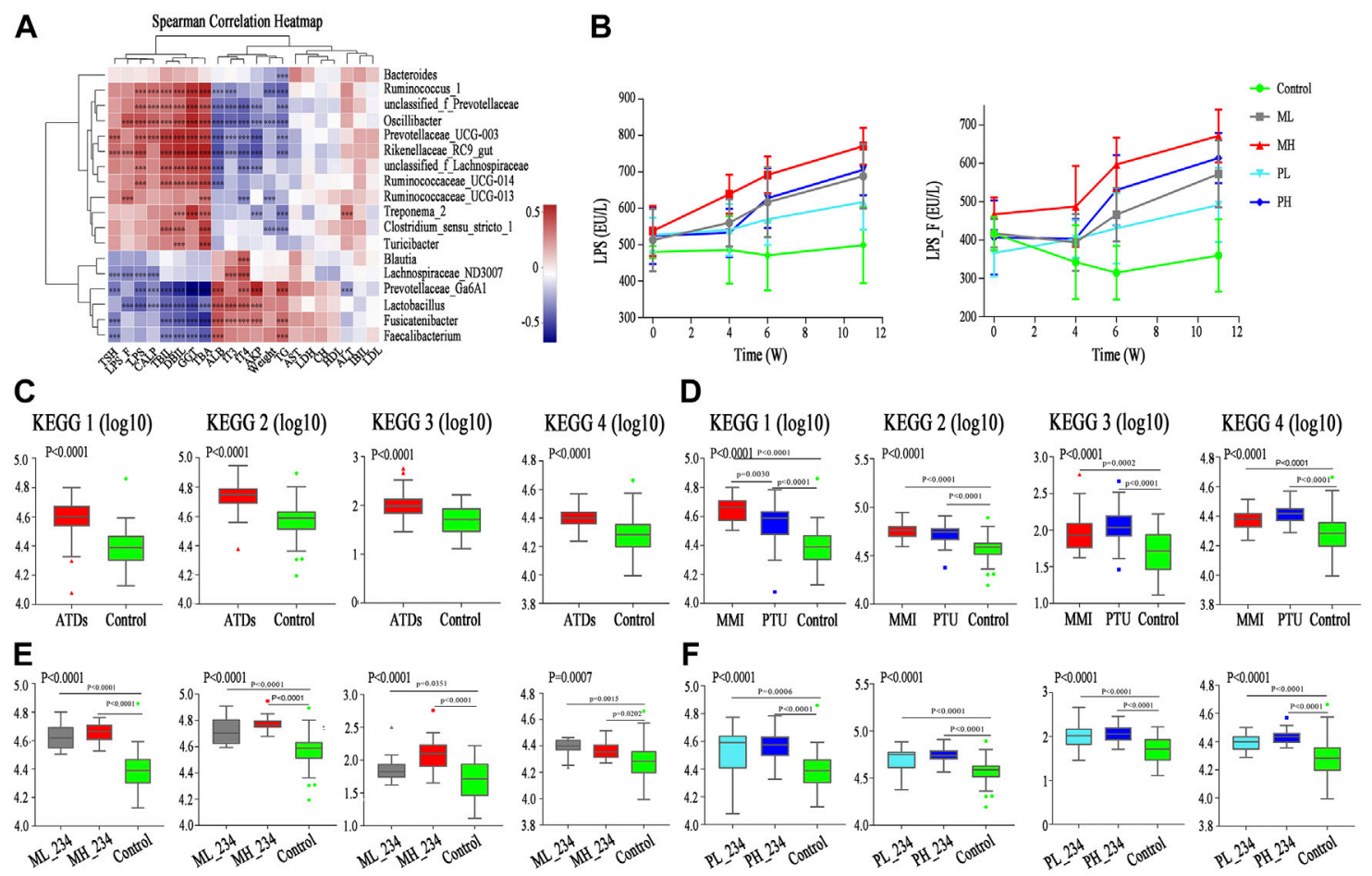

\section{$\mathbf{G}$}

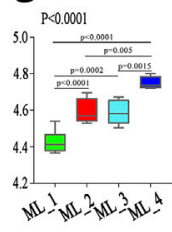

I

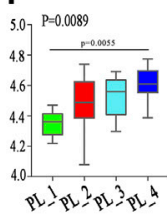

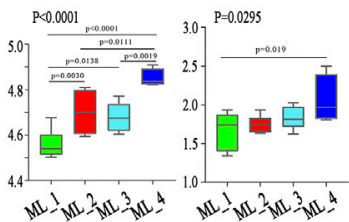

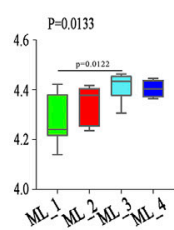

$\mathrm{H}$
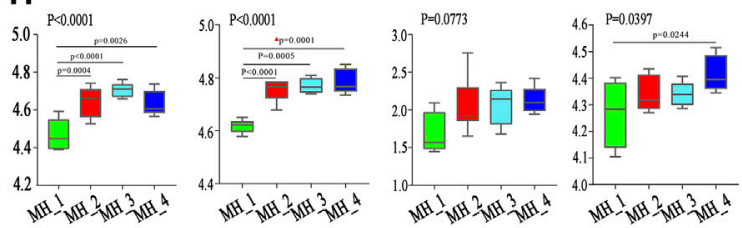

J
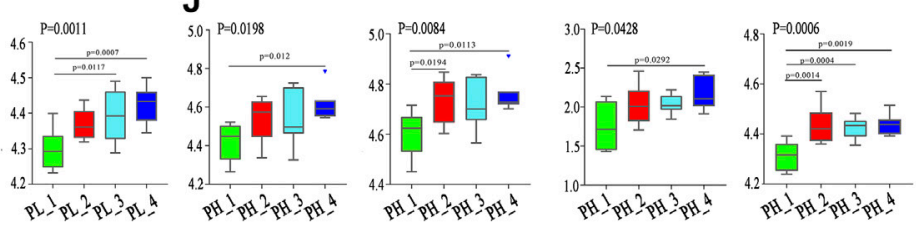

KEGG 1: Lipopolysaccharide biosynthesis KEGG 2: Lipopolysaccharide biosynthesis proteins KEGG 3: Bacterial invasion of epithelial cells KEGG 4: Bacterial toxins

$\mathbf{L}$
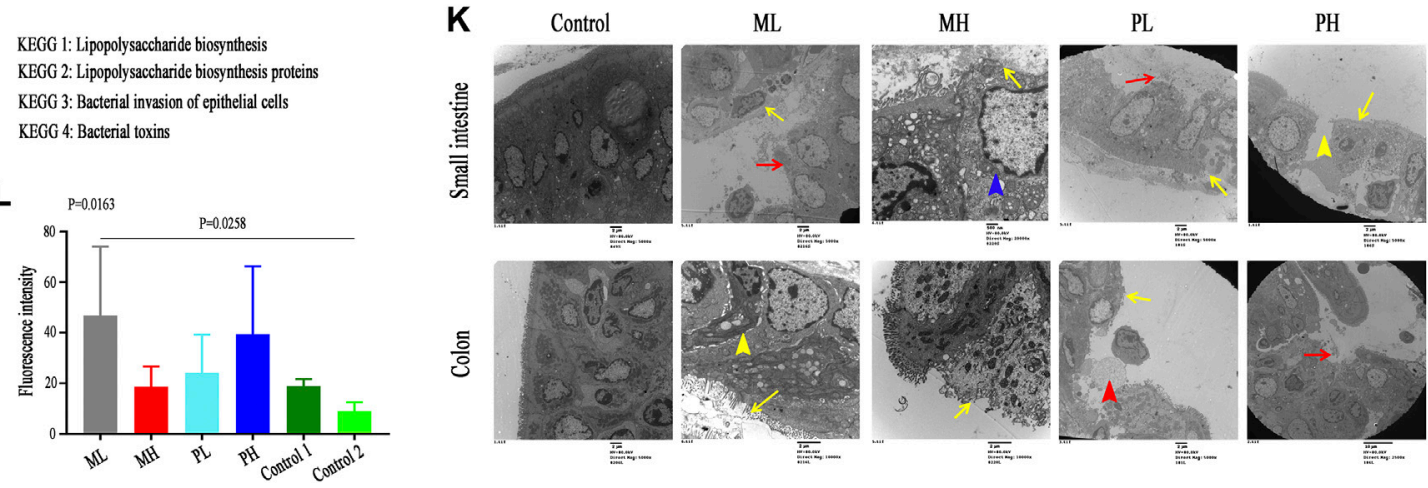

FIGURE 7 | Correlation between differential microbiota and basal indicators, and change of KEGG and gut barrier related indexes. (A) Spearman correlation analysis of 21 basal indicators and 18 differential genera; color intensity represents the magnitude of correlation. Red, positive correlations; blue, negative correlations. ${ }^{*} p$ value $<0.05 ;{ }^{* \star} p$ value $<0.01 ;{ }^{* \star *} p$ value $<0.001$. (B) LPS concentration in serum (LPS) and fecal supernatant (LPS_F) among subgroups. (C-J) The change of pathway Lipopolysaccharide biosynthesis, Lipopolysaccharide biosynthesis proteins, Bacterial invasion of epithelial cells, and Bacterial toxins among groups with ATDs, different ATDs, different doses and different time points. (K) Transmission electron microscopy analysis of small intestine (upper) and colon (lower) among different subgroups. Yellow long arrow means epithelial villus shedding; red long arrow means intestinal epithelium shedding; yellow short arrow means cell shrinkage; blue arrow means cell swelling and red short arrow means cell debris. (L) Serum FITC-dextran levels among different groups. 
accompanied by intestinal barrier destruction, which allows microbiota-derived LPS to enter the blood and triggers a series of downstream inflammatory reactions and organ damage. Additionally, the fecal CALP concentration, which reflects the intestinal inflammatory response, is also an index reflecting intestinal permeability and intestinal barrier, increased with ATDs administration (Wilbrink et al., 2020) (Supplementary Table S4). Therefore, we further investigated relevant intestinal barrier indicators.

\section{Changes in the Intestinal Barrier Structure and Function}

Transmission electron microscopy analysis of the control group showed that intestinal enterocytes presented regularly shaped mitochondria with intact cristae and microvilli that were thick, with lengths within the normal range (Figure 7K); colon enterocytes also presented regularly shaped mitochondria with intact cristae, and microvilli were neatly arranged but not as dense as those in the intestines. In the ML group, intestinal enterocytes showed only focal intestinal epithelial villus shedding and epithelial shedding; colon epithelial villus shedding was more severe than that in the intestines, some cells became pyknotic, and the intercellular space widened. $\mathrm{MH}$ group rats showed extensive epithelial villus shedding and mitochondrial swelling, and most of the colon epithelial villi were exfoliated. Similarly, in the PL group rats, intestinal enterocytes showed only focal intestinal epithelial villus shedding and epithelial shedding; colon enterocytes showed extensive exfoliated epithelial villi and some scattered cell debris. More severe damage appeared in the $\mathrm{PH}$ group, such as in the intestine, which showed a large area of epithelial villus exfoliation and cell rupture; most of the colon lacked a normal epithelial structure. These results all showed that 1) destruction was more severe in the colon than in the small intestine and that 2) the high-dose group experienced more severe effects than the low-dose group.

Consistent with the destruction of the intestinal barrier physical structure, ATDs group rats had significantly elevated serum FITC-dextran levels compared with those in the control group rats (Figure 7L). PH group rats showed higher serum FITC-dextran levels than those in the PL group rats, while interestingly, the ML group showed higher FITC-dextran levels than the $\mathrm{MH}$ group, which was consistent with the higher alpha diversity in the ML group than in the $\mathrm{MH}$ group.

\section{DISCUSSION}

In the present study, both clinical and animal studies showed that ATDs caused changes in the gut microbiota structure, and various liver function indexes, such as ALT and GGT, increased after ATDs administration. In addition, feces and serum LPS increased. The correlation between the disturbed gut microbiota structure, increased LPS level, and corresponding changes in clinical parameters indicate that the gut microbiota may mediate ATDs-induced liver injury through the intestinal endotoxemia hypothesis.
We focused mainly on the effect of ATDs on the gut microbiota structure. Although the differences in alpha diversity and beta diversity among subgroups in the clinical study were not always significant, they showed different variation tendencies. In the animal study, although the alpha diversity among different drug groups and most of the different dose groups showed no significant differences, nearly all the other alpha diversity and beta diversity indicators showed significant differences among the use or nonuse of ATDs, different ATDs, different doses and different time points, which reflect the fact that ATDs truly change the gut microbiota structure. The alpha diversity showed an increasing trend after ATDs administration in both the clinical and animal studies, which is contrary to the decrease of gut microbiota diversity caused by some drugs, such as antibiotics (Josefsdottir et al., 2017) and PPI (Jackson et al., 2016). This may due to intestinal bacterial overgrowth develops after recovery of hyperthyroidism in clinical study or hypothyroidism in animal study (Patil, 2014).

A differential gut microbiota analysis was also performed, and interestingly, the main changes in the microbiota in the clinical study differed from those in the animal study, as described above. For example, in the clinical study, ATDsinduced changes in the microbiota involved mainly Faecalibacterium, Eubacterium_rectale, Romboutsia, etc., while Lactobacillus, Romboutsia, unclassified_f_Prevotellaceae, etc., were changed primarily in the animal study. The abundance of some microbiota constituents, such as Romboutsia, increased in the clinical study but decreased in the animal study. These phenomena may be due to the differences between humans and rats. Furthermore, the clinical studies were based on the hyperthyroidism state in GD patients, whereas rats were evaluated under the ultimate hypothyroidism state. In addition, the clinical study also involved adjuvant drugs, such as hepatoprotective and leukogenic drugs. Though there were different gut microbiota constituents among clinical and animal studies, both showed that ATDs changed the gut microbiota and that different ATDs induced different gut microbiota structures. Furthermore, Faecalibacterium, which always reduced in some diseases such as Non-Alcoholic Fatty Liver Disease, showed low abundance both in clinical and animal experiments after ATDs administration (Zhou et al., 2018); unclassified_f_Lachnospiraceae, which was reported the key bacteria associated with inflammatory bowel disease patients or mouse model, also showed high abundance both in clinical and animal experiments after ATDs administration (Parada Venegas et al., 2019).

In addition, the gut microbiota and related metabolites all play important roles in maintaining homeostasis, and the same metabolites could be produced by different microbiotas. Recent studies have reported that SCFAs not only play an important role in regulating blood sugar and oxidizing energy supply but also are key in antipathogenic microorganisms for regulating intestinal microbiota balance, intestinal barrier function, systemic inflammation and hematopoiesis (Kim et al., 2014; Parada Venegas et al., 2019). In the present study, SCFAs-producing microbiota constituents, such as Faecalibacterium, Ruminococcaceae, Lactobacillus and Blautia, decreased after ATDs administration. 
The destruction of intestinal barrier could permit the translocation of LPS produced by intestinal gram-negative bacteria across the intestinal barrier and triggers systemic inflammation (Zhou et al., 2017). Gut microbiota dysbiosis and increasing intestinal permeability have been shown to be associated with many diseases, such as autism spectrum disorders (Strati et al., 2017) and nonalcoholic steatohepatitis (NASH) (Shen et al., 2017). In addition, the gut microbiota and the intestinal barrier participate in the side effects of some drugs. For example, oral iron therapies are administered for the management or improvement of iron deficiency anemia (IDA), although this treatment is often accompanied by intestinal and liver injury, which may be caused by gut microbiota and intestinal barrier destruction (Fang et al., 2018). Similarly, cyclophosphamide treatment, which exerts antitumor effects, is accompanied by side effects, such as intestinal injury, and a calorie-restricted diet protects the gut microbiota and intestinal barrier from injury (Liu et al., 2019). Antibiotics disturb the microbiota structure and intestinal barrier, sometimes accompanied by myelosuppression; and gut microbiota reconstruction by fecal microbiota transplantation can partially restore hematopoiesis (Josefsdottir et al., 2017). In the present study, both intestinal barrier structure and function were destroyed, according to transmission electron microscopy and FITC-dextran tests, after ATDs administration. In addition, fecal CALP content, which reflects the intestinal inflammatory state, was also increased in both the clinical and animal studies. In addition to the SCFAs-producing microbiota mentioned above, the abundance of Eubacterium_rectale, which is also considered an intestinal barrier-protecting microbiota, decreased, while microbiota that destroy the intestinal barrier, such as Dorea and Treponema_2, increased, thus promoting intestinal barrier destruction (Del Chierico et al., 2016; Xie et al., 2016).

Another key molecule, LPS, which is believed to originate from the gram-negative gut microbiome, could leak into the systemic circulation and stimulate proinflammatory cytokine expression, inducing organ system injury. In addition, excessive LPS production could directly destroy the intestinal barrier, which in turn permits LPS to pass through the leaky gut (Cani et al., 2008). Animal studies revealed that LPS administration to mice via oral gavage induced a dramatic reduction in ZO-1 expression at $24 \mathrm{~h}$, indicating intestinal barrier damage (Mouries et al., 2019). In the present study, the gut microbiota varied after ATDs exposure, and a characteristic change was the increase in the abundance of Enterobacteriaceae, which was thought to be the main LPS-producing microbiota (Vatanen et al., 2016) in the clinical study, especially in the MMI group. In an animal study, Enterobacteriaceae abundance also increased, although showing a lower abundance than that in the clinical study. In addition, analysis of microbiota functional alterations based on PICRUSt showed that the four LPS-related pathways were enriched after ATDs intervention compared with those in controls; the fecal and serum LPS levels in both the clinical and animal ATDs groups were higher than those in the control group, and the increase in LPS content was correlated with the change in blood indicators.

The bidirectional influence between the gut microbiota and drugs has long been studied. Broad-spectrum antibiotics induced gut microbiota dysbiosis which in turn is correlated with side effects, such as liver function abnormalities and hematopoietic depression, as mentioned above (Josefsdottir et al., 2017; Gong et al., 2018). Imhann et al. reported that long-term PPI use causes an increase in Enterobacteriaceae and a decrease in Ruminococcaceae abundances at the family level. These changes in symbiotic microbiota constituents increased susceptibility to Clostridium difficile infection (Jackson et al., 2016). ATDs also increased Enterobacteriaceae and decreased Ruminococcaceae abundances, and the increase in the Enterobacteriaceae abundance was correlated with intestinal barrier destruction, which could be reflected by the increase in fecal CALP content after the use of both PPIs and ATDs. Additionally, LPS and TLR-4 play important roles in the gastrointestinal toxicity effects of chemotherapeutic irinotecan (Wardill et al., 2016) and the LPS and related pathways also showed higher levels after ATDs administration than in the controls in both the clinical and animal studies.

In summary, the ATDs caused changes in the gut microbiota structure, and the gut microbiota in turn was correlated with side effects. Not all of the side effects involved liver injury or hematopoietic depression; however, the drug-induced side effects were associated with LPS production and intestinal barrier destruction, which is consistent with our results.

\section{CONCLUSION}

In the present study, we identified a close relationship among ATDs, gut microbiota dysbiosis, intestinal barrier destruction, LPS increase and liver injury. Gut microbiota dysbiosis was accompanied by intestinal barrier destruction, which induced increased LPS content entering the blood and triggering related liver injury. This study was the first to link the gut microbiota with ATDs-induced liver injury, which may provide new therapeutic targets and help reduce the liver injury of ATDs.

Inevitable limitations of our study must be mentioned. First, the clinical and animal experiments corresponded to two different thyroid function states, hyperthyroidism and hypothyroidism, which may lead to different gut microbiota changes. On the other hand, ATDs application must be accompanied by a change in thyroid function, and in vitro experiments would be unable to simulate the internal environment. Second, the clinical study involved the use of hepatoprotective drugs and leukogenic drugs, which may also play important roles in the gut microbiota structure. Third, this study did not conduct in-depth research on differentially abundant species, due mainly to the limitations of bacterial isolation culture techniques. Fourth, we performed a correlation analysis with no further intervention or mechanistic study, which need to be elucidated in the future.

\section{DATA AVAILABILITY STATEMENT}

The datasets presented in this study can be found in online repositories. The names of the repository/repositories and 
accession number can be found below: NCBI BioProject, accession no: PRJNA598313.

\section{ETHICS STATEMENT}

The studies involving human participants were reviewed and approved by Ethics Committee of the First Affiliated Hospital of Harbin Medical University. The patients/participants provided their written informed consent to participate in this study. The animal study was reviewed and approved by Ethics Committee of the First Affiliated Hospital of Harbin Medical University.

\section{AUTHOR CONTRIBUTIONS}

JS, FZ, and YW designed and supervised the project. JS, BL, and JF collected the samples. JS, FZ, and BL performed the bioinformatics and statistical analyses and interpreted the data. JS and FZ drafted the manuscript. YW revised the manuscript for important content.

\section{REFERENCES}

Aziz, R. K., Hegazy, S. M., Yasser, R., Rizkallah, M. R., and ElRakaiby, M. T. (2018). Drug pharmacomicrobiomics and toxicomicrobiomics: from scattered reports to systematic studies of drug-microbiome interactions. Expet Opin. Drug Metabol. Toxicol. 14, 1043-1055. doi:10.1080/17425255.2018.1530216

Bartalena, L., Burch, H. B., Burman, K. D., and Kahaly, G. J. (2016). A 2013 European survey of clinical practice patterns in the management of Graves' disease. Clin. Endocrinol. (Oxf) 84, 115-120. doi:10.1111/cen.12688

Brito, J. P., Schilz, S., Singh Ospina, N., Rodriguez-Gutierrez, R., Maraka, S., Sangaralingham, L. R., et al. (2016). Antithyroid drugs-the most common treatment for Graves' disease in the United States: a nationwide populationbased study. Thyroid 26, 1144-1145. doi:10.1089/thy.2016.0222

Cani, P. D., Bibiloni, R., Knauf, C., Waget, A., Neyrinck, A. M., Delzenne, N. M., et al. (2008). Changes in gut microbiota control metabolic endotoxemiainduced inflammation in high-fat diet-induced obesity and diabetes in mice. Diabetes 57, 1470-1481. doi:10.2337/db07-1403

Cani, P. D. (2018). Human gut microbiome: hopes, threats and promises. Gut 67, 1716-1725. doi:10.1136/gutjnl-2018-316723

Cano-Europa, E., Blas-Valdivia, V., Franco-Colin, M., Gallardo-Casas, C. A., and Ortiz-Butron, R. (2011). Methimazole-induced hypothyroidism causes cellular damage in the spleen, heart, liver, lung and kidney. Acta Histochem. 113, 1-5. doi:10.1016/j.acthis.2009.07.004

Chassaing, B., Etienne-Mesmin, L., and Gewirtz, A. T. (2014). Microbiota-liver axis in hepatic disease. Hepatology 59, 328-339. doi:10.1002/hep.26494

Chen, J., Wei, Y., He, J., Cui, G., Zhu, Y., Lu, C., et al. (2014). Natural killer T cells play a necessary role in modulating of immune-mediated liver injury by gut microbiota. Sci. Rep. 4, 7259. doi:10.1038/srep07259

Del Chierico, F., Nobili, V., Vernocchi, P., Russo, A., De Stefanis, C., Gnani, D., et al. (2016). Gut microbiota profiling of pediatric nonalcoholic fatty liver disease and obese patients unveiled by an integrated meta-omics-based approach. Hepatology 65, 451-464. doi:10.1002/hep.28572

Enright, E. F., Gahan, C. G., Joyce, S. A., and Griffin, B. T. (2016). The impact of the gut microbiota on drug metabolism and clinical outcome. Yale J. Biol. Med. 89, 375-382

Fang, S., Zhuo, Z., Yu, X., Wang, H., and Feng, J. (2018). Oral administration of liquid iron preparation containing excess iron induces intestine and liver injury, impairs intestinal barrier function and alters the gut microbiota in rats. J. Trace Elem. Med. Biol. 47, 12-20. doi:10.1016/j.jtemb.2018.01.002

\section{FUNDING}

This work was supported by the National Natural Science Foundation of China grants (81970466); 2017-2018 Special Fund for Scientific Research Transformation of Heilongjiang Academy of Medical Sciences (CR201803) and Project of scientific research and practical innovation for Postgraduates of Harbin Medical University (YJSKYCX2018-41HYD).

\section{ACKNOWLEDGMENTS}

We thank Baoli Ning, Xiaorong Zhan, Yanbo Li, Hongyu Kuang, Huijuan Zhang, Haoling Liu and Xinyuan Gao for their contribution to the donor screening.

\section{SUPPLEMENTARY MATERIAL}

The Supplementary Material for this article can be found online at: https://www.frontiersin.org/articles/10.3389/fphar.2020.598170/ full\#supplementary-material.

Franklyn, J. A. (2009). Thyroid gland: antithyroid therapy--best choice of drug and dose. Nat. Rev. Endocrinol. 5, 592-594. doi:10.1038/nrendo.2009.201

Fukui, M., Fukui, N., Sakai, K., Hasegawa, Y., Nagasaki, S., Shibata, S., et al. (2013). Spleen-specific development of germinal centers in rats treated with antithyroid drugs. J. Toxicol. Pathol. 26, 375-384. doi:10.1293/tox.2013-0012

Gevers, D., Kugathasan, S., Denson, L. A., Vazquez-Baeza, Y., Van Treuren, W., Ren, B., et al. (2014). The treatment-naive microbiome in new-onset crohn's disease. Cell Host Microbe 15, 382-392. doi:10.1016/j.chom.2014.02.005

Gong, S., Lan, T., Zeng, L., Luo, H., Yang, X., Li, N., et al. (2018). Gut microbiota mediates diurnal variation of acetaminophen induced acute liver injury in mice. J. Hepatol. 69, 51-59. doi:10.1016/j.jhep.2018.02.024

Gong, S., Yan, Z., Liu, Z., Niu, M., Fang, H., Li, N., et al. (2019). Intestinal microbiota mediates the susceptibility to polymicrobial sepsis-induced liver injury by granisetron generation in mice. Hepatology 69, 1751-1767. doi:10. 1002/hep.30361

Heidari, R., Niknahad, H., Jamshidzadeh, A., Eghbal, M. A., and Abdoli, N. (2015). An overview on the proposed mechanisms of antithyroid drugs-induced liver injury. Adv. Pharmaceut. Bull. 5, 1-11. doi:10.5681/apb.2015.001

Ishaq, H. M., Mohammad, I. S., Shahzad, M., Ma, C., Raza, M. A., Wu, X., et al. (2018). Molecular alteration analysis of human gut microbial composition in graves' disease patients. Int. J. Biol. Sci. 14, 1558-1570. doi:10.7150/ijbs. 24151

Jackson, M. A., Goodrich, J. K., Maxan, M. E., Freedberg, D. E., Abrams, J. A., Poole, A. C., et al. (2016). Proton pump inhibitors alter the composition of the gut microbiota. Gut 65, 749-756. doi:10.1136/gutjnl-2015-310861

Josefsdottir, K. S., Baldridge, M. T., Kadmon, C. S., and King, K. Y. (2017). Antibiotics impair murine hematopoiesis by depleting the intestinal microbiota. Blood 129, 729-739. doi:10.1182/blood-2016-03-708594

Kim, C. H., Park, J., and Kim, M. (2014). Gut microbiota-derived short-chain Fatty acids, T cells, and inflammation. Immune Netw. 14, 277-288. doi:10.4110/in. 2014.14.6.277

Kyriacou, A., Kyriacou, A., Makris, K. C., Syed, A. A., and Perros, P. (2019). Weight gain following treatment of hyperthyroidism-A forgotten tale. Clin Obes 9, e12328. doi: $10.1111 /$ cob.12328

Li, C., Tan, J., Zhang, G., Meng, Z., Wang, R., Li, W., et al. (2015). Risk factors of hyperthyroidism with hepatic function injury: a 4-year retrospective study. Horm. Metab. Res. 47, 209-213. doi:10.1055/s-0034-1375690

Li, H., He, J., and Jia, W. (2016). The influence of gut microbiota on drug metabolism and toxicity. Expet Opin. Drug Metabol. Toxicol. 12, 31-40. doi:10.1517/17425255.2016.1121234 
Li, H., and Jia, W. (2013). Cometabolism of microbes and host: implications for drug metabolism and drug-induced toxicity. Clin. Pharmacol. Ther. 94, 574-581. doi:10.1038/clpt.2013.157

Liu, T., Wu, Y., Wang, L., Pang, X., Zhao, L., Yuan, H., et al. (2019). A more robust gut microbiota in calorie-restricted mice is associated with attenuated intestinal injury caused by the chemotherapy drug cyclophosphamide. mBio 10, e0290318. doi:10.1128/mBio.02903-18

Maier, L., Pruteanu, M., Kuhn, M., Zeller, G., Telzerow, A., Anderson, E. E., et al. (2018). Extensive impact of non-antibiotic drugs on human gut bacteria. Nature 555, 623-628. doi:10.1038/nature25979

Manzo, V. E., and Bhatt, A. S. (2015). The human microbiome in hematopoiesis and hematologic disorders. Blood 126, 311-318. doi:10.1182/blood-2015-04-574392

Masetti, G., Moshkelgosha, S., Köhling, H. L., Covelli, D., Banga, J. P., Berchnerpfannschmidt, U., et al. (2018). Gut microbiota in experimental murine model of Graves' orbitopathy established in different environments may modulate clinical presentation of disease. Microbiome 6, 97. doi:10.1186/ s40168-018-0478-4

Meng, X., Li, S., Li, Y., Gan, R. Y., and Li, H. B. (2018). Gut microbiota's relationship with liver disease and role in hepatoprotection by dietary natural products and probiotics. Nutrients 10, 1457. doi:10.3390/nu10101457

Montassier, E., Al-Ghalith, G. A., Ward, T., Corvec, S., Gastinne, T., Potel, G., et al. (2016). Pretreatment gut microbiome predicts chemotherapy-related bloodstream infection. Genome Med. 8, 49. doi:10.1186/s13073-016-0301-4

Morgan, A. P., Crowley, J. J., Nonneman, R. J., Quackenbush, C. R., Miller, C. N., Ryan, A. K., et al. (2014). The antipsychotic olanzapine interacts with the gut microbiome to cause weight gain in mouse. PLoS One 9, e115225. doi:10.1371/ journal.pone. 0115225

Mouries, J., Brescia, P., Silvestri, A., Spadoni, I., Sorribas, M., Wiest, R., et al. (2019). Microbiota-driven gut vascular barrier disruption is a prerequisite for nonalcoholic steatohepatitis development. J. Hepatol. 71, 1216-1228. doi:10.1016/j. jhep.2019.08.005

Parada Venegas, D., De la Fuente, M. K., Landskron, G., Gonzalez, M. J., Quera, R., Dijkstra, G., et al. (2019). Short chain fatty acids (SCFAs)-Mediated gut epithelial and immune regulation and its relevance for inflammatory bowel diseases. Front. Immunol. 10, 277. doi:10.3389/fimmu.2019.00277

Patil, A. D. (2014). Link between hypothyroidism and small intestinal bacterial overgrowth. Indian J. Endocrinol. Metab. 18, 307-309. doi:10.4103/2230-8210.131155

Romano, K. A., Vivas, E. I., Amador-Noguez, D., and Rey, F. E. (2015). Intestinal microbiota composition modulates choline bioavailability from diet and accumulation of the proatherogenic metabolite trimethylamine- $\mathrm{N}$-oxide. mBio 6, e02481. doi:10.1128/mBio.02481-14

Russo, M. W., Galanko, J. A., Shrestha, R., Fried, M. W., and Watkins, P. (2004). Liver transplantation for acute liver failure from drug induced liver injury in the united states. Liver Transpl. 10, 1018-1023. doi:10.1002/lt.20204

Shen, F., Zheng, R. D., Sun, X. Q., Ding, W. J., Wang, X. Y., and Fan, J. G. (2017). Gut microbiota dysbiosis in patients with non-alcoholic fatty liver disease. Hepatobiliary Pancreat. Dis. Int. 16, 375-381. doi:10.1016/S1499-3872(17)60019-5

Shen, T., Liu, Y., Shang, J., Xie, Q., Li, J., Yan, M., et al. (2019). Incidence and etiology of drug-induced liver injury in mainland china. Gastroenterology 156, 2230-2241.e11. doi:10.1053/j.gastro.2019.02.002

Shi, T. T., Xin, Z., Hua, L., Zhao, R. X., Yang, Y. L., Wang, H., et al. (2019). Alterations in the intestinal microbiota of patients with severe and active Graves' orbitopathy: a cross-sectional study. J. Endocrinol. Invest. 42, 967-978. doi:10.1007/s40618-019-1010-9

Shibutani, M., Woo, G. H., Fujimoto, H., Saegusa, Y., Takahashi, M., Inoue, K., et al. (2009). Assessment of developmental effects of hypothyroidism in rats from in utero and lactation exposure to anti-thyroid agents. Reprod. Toxicol. 28, 297-307. doi:10.1016/j.reprotox.2009.04.011

Strati, F., Cavalieri, D., Albanese, D., De Felice, C., Donati, C., Hayek, J., et al. (2017). New evidences on the altered gut microbiota in autism spectrum disorders. Microbiome 5, 24. doi:10.1186/s40168-017-0242-1

Turnbaugh, P. J., Ley, R. E., Mahowald, M. A., Magrini, V., Mardis, E. R., and Gordon, J. I. (2006). An obesity-associated gut microbiome with increased capacity for energy harvest. Nature 444, 1027-1031. doi:10.1038/nature05414

Vatanen, T., Kostic, A. D., d'Hennezel, E., Siljander, H., Franzosa, E. A., Yassour, M., et al. (2016). Variation in microbiome LPS immunogenicity contributes to autoimmunity in humans. Cell 165, 842-853. doi:10.1016/j.cell.2016.04.007

Wang, M. T., Lee, W. J., Huang, T. Y., Chu, C. L., and Hsieh, C. H. (2014). Antithyroid drug-related hepatotoxicity in hyperthyroidism patients: a population-based cohort study. Br. J. Clin. Pharmacol. 78, 619-629. doi:10. $1111 /$ bcp. 12336

Wardill, H. R., Gibson, R. J., Van Sebille, Y. Z., Secombe, K. R., Coller, J. K., White, I. A., et al. (2016). Irinotecan-induced gastrointestinal dysfunction and pain are mediated by common TLR4-dependent mechanisms. Mol. Cancer Ther. 15, 1376-1386. doi:10.1158/1535-7163.MCT-15-0990

Wilbrink, J., Bernards, N., Mujagic, Z., van Avesaat, M., Pijls, K., Klaassen, T., et al. (2020). Intestinal barrier function in morbid obesity: results of a prospective study on the effect of sleeve gastrectomy. Int. J. Obes. (Lond) 44, 368-376. doi:10.1038/s41366-019-0492-z

Wu, H., Esteve, E., Tremaroli, V., Khan, M. T., Caesar, R., Manneras-Holm, L., et al. (2017). Metformin alters the gut microbiome of individuals with treatmentnaive type 2 diabetes, contributing to the therapeutic effects of the drug. Nat. Med. 23, 850-858. doi:10.1038/nm.4345

Xie, G., Wang, X., Liu, P., Wei, R., Chen, W., Rajani, C., et al. (2016). Distinctly altered gut microbiota in the progression of liver disease. Oncotarget 7 , 19355-19366. doi:10.18632/oncotarget.8466

Zarrinpar, A., Chaix, A., Xu, Z. Z., Chang, M. W., Marotz, C. A., Saghatelian, A., et al. (2018). Antibiotic-induced microbiome depletion alters metabolic homeostasis by affecting gut signaling and colonic metabolism. Nat. Commun. 9, 2872. doi:10.1038/s41467-018-05336-9

Zhou, D., Pan, Q., Xin, F. Z., Zhang, R. N., He, C. X., Chen, G. Y., et al. (2017). Sodium butyrate attenuates high-fat diet-induced steatohepatitis in mice by improving gut microbiota and gastrointestinal barrier. World J. Gastroenterol. 23, 60-75. doi:10.3748/wjg.v23.i1.60

Zhou, L., Li, X., Ahmed, A., Wu, D., Liu, L., Qiu, J., et al. (2014). Gut microbe analysis between hyperthyroid and healthy individuals. Curr. Microbiol. 69, 675-680. doi:10.1007/s00284-014-0640-6

Zhou, Y., Zheng, T., Chen, H., Li, Y., Huang, H., Chen, W., et al. (2018). Microbial intervention as a novel target in treatment of non-alcoholic fatty liver disease progression. Cell. Physiol. Biochem. 51, 2123-2135. doi:10.1159/000495830

Conflict of Interest: The authors declare that the research was conducted in the absence of any commercial or financial relationships that could be construed as a potential conflict of interest.

Copyright $\odot 2020$ Sun, Zhao, Lin, Feng, Wu, Liu, Zhao, Zhu and Wei. This is an open-access article distributed under the terms of the Creative Commons Attribution License (CC BY). The use, distribution or reproduction in other forums is permitted, provided the original author(s) and the copyright owner(s) are credited and that the original publication in this journal is cited, in accordance with accepted academic practice. No use, distribution or reproduction is permitted which does not comply with these terms. 